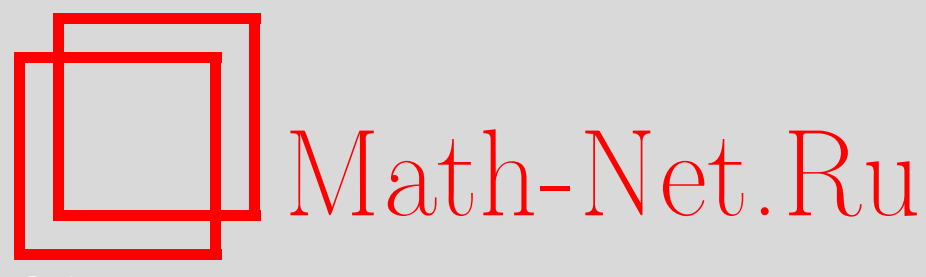

В. Де Альфаро, А. Т. Филиппов, Размерная редукция гравитации и связь между статическими состояниями, космологическими моделями и волнами, ТМФ, 2007, том 153, номер 3, 422-452

DOI: https://doi.org/10.4213/tmf6146

Использование Общероссийского математического портала Math-Net.Ru подразумевает, что вы прочитали и согласны с пользовательским соглашением http: //www . mathnet.ru/rus/agreement

Параметры загрузки:

IP : 35.173 .219 .12

26 апреля 2023 г., 16:51:46

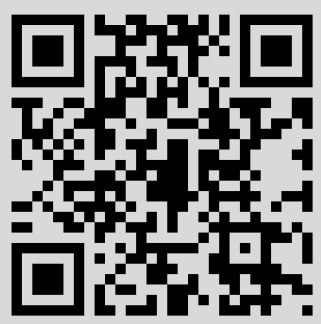




\title{
РАЗМЕРНАЯ РЕДУКЦИЯ ГРАВИТАЦИИ И СВЯЗЬ МЕЖДУ СТАТИЧЕСКИМИ СОСТОЯНИЯМИ, КОСМОЛОГИЧЕСКИМИ МОДЕЛЯМИ И ВОЛНАМИ
}

\begin{abstract}
Для интегрируемой $(1+1)$-мерной дилатонной гравитации, взаимодействующей с материей, введены обобщенные размерные редукции к одномерным статическим состояниям (в частности, черным дырам), космологическим моделям и волнам. Необычным свойством этих редукций оказывается то, что волновые решения зависят от двух переменных - пространства и времени. Решения можно получить как с помощью редукции пространства модулей (возможной ввиду полной интегрируемости), так и с помощью процедуры обобщенного разделения переменных (применимой также к неинтегрируемым моделям и к теориям в старших размерностях). Среди таких новых волноподобных решений обнаружен класс решений, в которых поля материи конечны всюду в пространстве-времени, включая бесконечность. Эти рассуждения явно показывают, что существует глубокая связь между статическими состояниями, космологиями и волнами. Приведены соображения в пользу того, что подобные связи могут иметь место также и в реалистичных теориях высших размерностей. Среди прочего вкратце указаны соотношения, существующие между теориями низших размерностей и реалистичными теориями старших размерностей.
\end{abstract}

Ключевые слова: дилатонная гравитация, размерная редукция, космология, интегрируемые модели, разделение переменных, гравитационные волны, супергравитация.

\section{1. ВВЕДЕНИЕ}

Последние наблюдения, свидетельствующие об ускорении расширения нашей Вселенной, приводят к необходимости рассмотрения более широкого класса космологических моделей, которые, возможно, смогут объяснить происхождение темной энергии. (Общий обзор проблемы темной энергии см. в работе [1]. Разнообразные космологические модели, пытающиеся объяснить происхождение темной энергии,

${ }^{*}$ Dipartmento di Fisica Teorica, Universita degli studi di Torino, and INFN, Torino, Italia; Accademia delle Scienze di Torino, Torino, Italia. E-mail: vda@to.infn.it

${ }^{\dagger}$ Объединенный институт ядерных исследований, г. Дубна, Московская обл., Россия. E-mail: filippov@theor.jinr.ru 
также описаны в работах [2], [3].) Хотя модельно-независимый анализ имеющихся экспериментальных данных [4] не противоречит самому простому из возможных усовершенствований, в котором эйнштейновская гравитация дополняется эйнштейновским же постоянным космологическим членом, это не проясняет ни физическое, ни геометрическое происхождение темной энергии. Многочисленные попытки понять происхождение темной энергии так или иначе связаны с оценкой роли различных скалярных полей материи в теории гравитации и роли (не обязательно постоянного) космологического потенциала. Такие поля могут возникать при размерных редукциях теорий гравитации или супергравитации, связанных с суперструнными теориями (см. общие обзоры космологических моделей, основанных на струнных теориях в период до темной энергии, например, в работах [5], [6]).

Можно также отметить, что полное понимание непертурбативных свойств даже стандартной гравитации, взаимодействующей с материей, все еще до конца не достигнуто, и эти свойства заслуживают тщательного исследования в полностью интегрируемых моделях гравитации, взаимодействующей с полями материи. Усилиями многих авторов в течение длительного времени были найдены и исследованы многочисленные точные решения гравитации без полей материи (см. достаточно полный обзор в работе [7]), а в более позднее время были открыты интегрируемые полевые модели, описывающие некоторые важные свойства теории гравитации (см., например, работы [8]-[11] и ссылки в них). Первая модель, содержащая некоторые наиболее важные свойства черных дыр, взаимодействующих со многими полями скалярной материи, появилась 15 лет назад [12] и пользовалась успехом, поскольку позволила понять некоторые особенности эволюции черных дыр. В космологии обычно исследовались только одномерные модели, а их связь с двумерными интегрируемыми моделями гравитации не обсуждалась. Возможное объяснение этого состоит в том, что модель Каллана-Гиддингса-Харви-Стромингера, возникающая в струнном подходе [12], оказывается недостаточно реалистичной, так как в ней взаимодействие со скалярными полями имеет самый простой характер, а космологический потенциал постоянен (и не соответствует космологической постоянной в старших размерностях). В результате геометрия двумерного пространства-времени в указанной модели оказывается тривиальной, а волны и космологии - совершенно нереалистичными. Гораздо более реалистичные двумерные модели были получены из сферически-симметричной гравитации, взаимодействующей с полями материи, но эти модели не интегрируемы. Некоторые реалистичные двумерные космологические модели также были инспирированы струнной теорией, и некоторые из них оказались связанными с интегрируемыми $\sigma$-моделями (см., например, [6]). К сожалению, эти модели не так хорошо подходят для описания черных дыр и прочих реалистичных статических состояний, поскольку в этих моделях космологический потенциал тождественно равен нулю.

Таким образом, имеются серьезные основания для изучения более сложных дву- 
мерных моделей гравитации, взаимодействующей с полями материи, и для сравнения этих моделей с более реалистичными приближениями, способными описать настоящие черные дыры, космологии и волны. В этой работе мы продолжаем исследование размерно редуцированных описаний теорий бран высших размерностей (в частности, черных дыр и других статических состояний), космологических теорий и некоторых волн, взаимодействующих с гравитацией, которое развивалось в работах [13]-[18]. Первая цель настоящей работы состоит в том, чтобы проиллюстрировать различные задачи и подходы к их решению на примере явно интегрируемой $N$-лиувиллевой модели гравитации, взаимодействующей с материей. Эта модель близка к некоторым реалистичным теориям и задает описание настоящих черных дыр и космологий в размерности 1.

Вторая цель заключается в демонстрации того, что наиболее общий подход к размерной редукции размерности $1+1$ к размерностям $1+0$ или $0+1$ может быть основан на правильно сформулированой процедуре разделения переменных. Также мы попытаемся более явно показать глубокую взаимосвязь, которая существует между черными дырами (или, более широко, между статическими состояниями гравитации, взаимодействующей с материей), различными космологическими моделями и, возможно, простейшими волнами скалярной материи, взаимодействующей с гравитацией.

Некоторое время назад нами было обнаружено, что наивная размерная редукция $(1+1)$-мерной дилатонной гравитации к одномерной не приводит к стандартной космологии Фридмана-Робертсона-Уокера (ФРУ). Это было отмечено в нашей работе [13], где мы сочли необходимым вернуться к исходной сферически-симметричной многомерной гравитации в высшей размерности (эквивалентной $(1+1)$-размерной теории) и непосредственно редуцировать ее к однородным и изотропным ФРУ-космологиям. Это представляется странным, так как ФРУ-космологии сферическисимметричны, а $(1+1)$-мерная дилатонная гравитация описывает все возможные сферически-симметричные решения исходной многомерной теории. Таким образом, вопрос, как получить стандартную космологию непосредственно из дилатонной гравитации с помощью некоторой размерной редукции, остался открытым. Наибольшим образом вводит в заблуждение тот факт, что наивная редукция порождает правильное статическое состояние чистой гравитации, т.е. внешнюю область шварцшильдовской черной дыры. При этом наивная космология - это просто внутренняя область черной дыры, которая не имеет ничего общего с ФРУ-космологией. Наличие полей материи существенно меняет картину, поскольку вместо черной дыры при этом возникает статическое состояние материи, не имеющее горизонта, а соответствующая космология оказывается одной из ФРУ-космологий (на самом деле, одной из замкнутых космологий). Этот результат был получен в давней работе [19], но никто (включая автора) не оценил его парадоксального значения. Заметим, что связь между шварцшильдовской черной дырой и некоторой космоло- 
гией была сначала отмечена в работе [20]. Эта связь неявно отмечалась в работах [21]. Более общий пример, демонстрирующий тесную связь между статическими сферически-симметричными состояниями скалярной материи, взаимодействующей с гравитацией, и сферически-симметричными замкнутыми космологиями, был приведен в работе [19], хотя его значение и не обсуждалось в деталях. В то же время были найдены сходные соотношения между некоторыми статическими $p$-бранами и космологиями, см. [22]-[24].

В попытке понять эти соотношения в работе [14] нами было предложено получить ФРУ-космологию с помощью более общей размерной редукции, в которой метрика и дилатон эффективно одномерных черных дыр и космологий зависят от двух переменных. Хотя в этом рассмотрении все еще использовалась связь двумерной метрики и метрики старших размерностей, нами было сделано заключение о возможном существовании непосредственных размерных редукций двумерной теории к ее одномерным потомкам. Еще один вывод говорит о возможности существования разнообразных обобщенных размерных редукций, порождающих различные статические и космологические решения, которые оказываются эффективно одномерными, но формально зависят от двух переменных.

Мимоходом отметим, что, насколько нам известно, эта задача никогда не обсуждалась в литературе. Возможное объяснение этого может состоять в том, что в течение многих лет исследования черных дыр и космологий следовали существенно различным подходам. Тот хорошо известный факт, что внутренняя часть шварцшильдовской черной дыры представляет собой некоторую космологию, рассматривался как курьез, не имеющий серьезного значения. В то время как теория черных дыр стремилась понять все более и более сложные теоретические модели (от сферически-симметричных коллапсирующих черных дыр до вращающихся аксиально-симметричных), в основе космологической теории лежала ФРУ-модель, и ведущее направление в космологии было связано с астрофизическими и наблюдательными аспектами и их адаптацией к современной физике. В настоящее время это настроение меняется в связи с исследованиями проблемы темной энергии, что, по-видимому, обеспечивает достаточную мотивацию поисков общей основы для всех трех главных объектов современной теории гравитации: статических состояний (в частности, черных дыр), космологических моделей и гравитационных волн (или волн материи, взаимодействующей с гравитацией).

В неопубликованной работе [15] была сделана попытка некоторым образом систематизировать отмеченные выше соображения. Например, была изучена идея, что нельзя отбрасывать "поверхностные" члены (или “полные производные" в лагранжиане), которые появляются в процессе размерной редукции. Также было найдено, что некоторые стандартные фиксации "калибровки" ("координатные" калибровки) могут быть опасными в этом контексте, поскольку они существенно ограничивают число возможных размерных редукций. Тем не менее проблема в целом оставалась 
непонятой. Например, в работе [15] нами все еще рассматривались лишь наивные размерные редукции интегрируемой $N$-лиувиллевой теории, т.е. фактически мы "нашли, но в упор не видели" (образно выражаясь) новый класс эффективно одномерных решений этой теории.

В работе [16] было отмечено, что структура решений интегрируемой $N$-лиувиллевой теории допускает очень простую интерпретацию размерной редукции как редукцию в пространстве модулей. Это немедленно приводит к тому, что в дополнение к статическим и космологическим решениям существует целый класс волноподобных решений. Попытка произвести более детальное исследование этих состояний предпринята нами в работе [18], которая не была опубликована в основном потому, что подход этой работы представлялся применимым исключительно к интегрируемым моделям.

В данной статье мы переписываем работу [18] с самого начала в свете результатов работ [16] и [17] и связываем “динамическую размерную редукцию” интегрируемых моделей с более общими редукциями, основанными на процедуре обобщенного разделения переменных (которая может быть в принципе применима и к неинтегрируемым теориям). Мы также исследуем в некоторых деталях волноподобные решения, которые могут быть получены в обоих подходах, и обсуждаем некоторые несингулярные волны скалярной материи, взаимодействующей с гравитацией.

\section{2. ОБЩИЕ СООТНОШЕНИЯ МЕЖДУ ДВУМЕРНЫМИ И ОДНОМЕРНЫМИ ЛАГРАНЖИАНАМИ}

Рассмотрим двумерный лагранжиан достаточно общего вида

$$
\mathcal{L}^{(2)}=\sqrt{-g}\left\{\varphi R+V(\varphi, \psi)+\sum_{m=3}^{N} Z_{m n}(\varphi, \psi) \nabla \psi_{m} \nabla \psi_{n}\right\}
$$

где $g_{i j}$ представляет собой общую $(1+1)$-мерную метрику с сигнатурой $(-1,1)$, $\varphi$ - дилатонное поле, $R$ - кривизна Риччи, $V(\varphi, \psi)$ - эффективный ("космологический") потенциал, а матрица $Z_{m n}(\varphi, \psi)$ взаимодействия скалярных полей является произвольной функцией от дилатонных полей и от $(N-2)$ скалярных безмассовых полей $\psi_{n}{ }^{1)}$. Эта теория оказывается интегрируемой в двух основных случаях: во-первых, когда $V \equiv 0$ и, во-вторых, когда все $Z_{m n}$ являются константами, не зависящими от полей, а $V$ представляет собой специально выбранный потенциал

\footnotetext{
1) В моделях (1), возникающих при размерных редукциях из теорий (супер)гравитации старших размерностей, матрица взаимодействия $Z_{m n}$ вещественна, симметрична и невырожденна. Будем также предполагать, что все ее собственные значения отрицательны. От последнего ограничения можно отказаться, если ввести так называемые "фантомные" поля, но такие экзотические модели остаются за рамками нашего рассмотрения. Обычно присутствующий дилатонный кинетический член $W(\nabla \varphi)^{2}$ можно исключить из лагранжиана с помощью преобразований Вейля, так что здесь мы его опускаем (см. [14], [16]).
} 
(см. [16], [17]). Если все собственные значения матрицы $Z_{m n}$ отрицательны, то поля $\psi_{n}$ можно в последнем случае преобразовать так, чтобы

$$
Z_{m n}=-\delta_{m n}
$$

Остановимся на этом случае и будем считать, что потенциал $V(\varphi, \psi)$ имеет вид

$$
V=\sum_{n=1}^{N} g_{n} e^{q_{n}^{(0)}}, \quad q_{n}^{(0)} \equiv a_{n} \varphi+\sum_{m=3}^{N} \psi_{m} a_{m n} .
$$

Теория интегрируема, если параметры $a_{n}, a_{n m}$ удовлетворяют условию

$$
\sum_{l=3}^{N} a_{l m} a_{l n}-2\left(a_{m}+a_{n}\right)=\gamma_{n}^{-1} \delta_{m n},
$$

а $Z_{m n}$ удовлетворяет (2). При $m \neq n$ условие (4) представляет собой условие псевдоортогональности на $N$-векторы $A_{n} \equiv\left\{a_{m n}\right\}, m=1, \ldots, N, a_{1 n} \equiv 1+a_{n}, a_{2 n} \equiv 1-a_{n}$, т.е. (4) эквивалентно уравнению

$$
A_{n} \cdot A_{m} \equiv-a_{1 m} a_{1 m}+\sum_{l=2}^{N} a_{l m} a_{l n}=0 .
$$

При $m=n$ равенство (4) задает $\gamma_{n}$ - важные параметры теории,

$$
\gamma_{n}^{-1}=A_{n} \cdot A_{n}=-4 a_{n}+\sum_{l=3}^{N} a_{l n}^{2} .
$$

Детальное описание свойств параметров $a_{m n}, \gamma_{n}$, удовлетворяющих (4), см. в работах [14]-[17] (в них было показано, что одна и только одна норма среди $\gamma_{n}^{-1}$ является отрицательной; обычно выбирается $\left.\gamma_{1}<0\right)$.

Заметим, что интегрируемость не зависит от значений $g_{n}$ констант связи. Некоторые из них могут даже обращаться в нуль ${ }^{2)}$. Также без изменения свойства интегрируемости к теории (1) можно добавить произвольное число свободных безмассовых скалярных полей. Во всех этих случаях общее решение двумерной интегрируемой теории, определяемой уравнениями (1)-(4), можно выписать явно.

В настоящей работе упор делается на размерной редукции $(1+1)$-мерной интегрируемой модели к интегрируемой одномерной модели. Перед тем как обратиться к основным рассуждениям, укажем, как эти интегрируемые модели соотносятся с реалистичными теориями. Для упрощения представления будем в основном работать в (конформно плоской) калибровке светового конуса (CK), в которой метрика имеет вид

$$
d s^{2}=-4 f(u, v) d u d v \equiv-4 \varepsilon e^{F(u, v)} d u d v=e^{F}\left(d r^{2}-d t^{2}\right), \quad \varepsilon= \pm 1,
$$

${ }^{2)}$ Если теория (1) получается с помощью размерной редукции многомерной теории супергравитации на торе, константы связи $g_{n}$ должны быть отрицательными. Исключение составляет случай сферически-симметричной редукции, которая приводит к появлению одного положительного члена в двумерном потенциале (3). 
с параметрами $r$ и $t$, определяемыми как $r \equiv u-\varepsilon v, t \equiv u+\varepsilon v$. В экспоненциальном представлении метрики в (7) нули функции $f(u, v)$ (горизонты) могут появиться при $F \rightarrow-\infty$. При пересечении горизонта происходит перемена знака $f$, определяющаяся значением $\varepsilon$, но представление пространства-времени остается неизменным ввиду автоматического изменения соотношения между координатами пространства-времени и СК (что приводит к взаимной замене $r \leftrightarrow t$ ).

Наивная редукция к размерности 1 (пространственной или временно́й) получится, если предположить, что метрика, дилатон и поля скалярной материи зависят от единственной переменной $\tau=a(u)+b(v)$, которая может быть либо пространственной, либо временно́й. Редуцированный лагранжиан принимает при этом вид

$$
\mathcal{L}^{(1)}=-\frac{1}{l(\tau)}\left(\dot{\varphi} \dot{F}+\sum_{m, n=3}^{N} Z_{m n} \dot{\psi}_{m} \dot{\psi}_{n}\right)+l(\tau) \varepsilon e^{F} V(\varphi, \psi) .
$$

Если $Z_{m n}=-\delta_{m n}$, то уравнения $(3),(4)$ задают также достаточное условие интегрируемости.

Однако теория (8) может оказаться интегрируемой и в других случаях. Например, предположим, что $Z_{m n}=-\left(1 / \phi^{\prime}(\varphi)\right) \delta_{m n}$, где $\phi^{\prime}(\varphi)>0$. Тогда

$$
\mathcal{L}^{(1)}=\frac{1}{l(\tau) \phi^{\prime}(\phi)}\left(-\dot{\phi} \dot{F}+\sum_{n=3}^{N} \dot{\psi}_{n}^{2}\right)+\left[l(\tau) \phi^{\prime}(\varphi)\right] \varepsilon e^{F}\left[\frac{V}{\phi^{\prime}(\varphi)}\right] .
$$

Вводя новый лагранжев множитель $\bar{l}(\tau) \equiv l(\tau) \phi^{\prime}(\varphi)$ и полагая

$$
\frac{V(\varphi, \psi)}{\phi^{\prime}(\varphi)} \equiv \bar{V}(\phi, \psi)
$$

получим, что новый одномерный лагранжиан запишется как

$$
\overline{\mathcal{L}}^{(1)}=\frac{1}{\bar{l}(\tau)}\left(-\dot{\phi} \dot{F}+\sum_{n=3}^{N} \dot{\psi}_{n}^{2}\right)+\bar{l}(\tau) \varepsilon e^{F} \bar{V}(\phi, \psi) .
$$

Эта модель интегрируема, если новый потенциал $\bar{V}(\phi, \psi)$ можно записать в виде (3) с параметрами $\bar{a}_{n}, a_{m n}(m \geqslant 3)$, удовлетворяющими условиям ортогональности (4):

$$
\begin{gathered}
\bar{V}(\phi, \psi)=\sum_{n=1}^{N} g_{n} e^{\bar{q}_{n}^{(0)}}, \quad \bar{q}_{n}^{(0)}=\bar{a}_{n} \phi+\sum_{m=3}^{N} \psi_{m} a_{m n}, \\
\sum_{l=3}^{N} a_{l m} a_{l n}-2\left(\bar{a}_{m}+\bar{a}_{n}\right)=\bar{\gamma}_{n}^{-1} \delta_{m n} .
\end{gathered}
$$

Интегрируемая одномерная модель (8) получена из интегрируемой двумерной модели (1), причем обе эти модели удовлетворяют условиям интегрируемости (2)-(4). В отличие от модели (8) новая интегрируемая модель (10) получается из заведомо неинтегрируемой двумерной теории. В самом деле, чтобы получить потенциал $\bar{V}$, 
экспоненциально зависящий от поля $\phi$, следует начинать с потенциала $V$, который неэкспоненциален относительно поля $\varphi$ и содержит зависящие от $\varphi$ константы связи $Z_{m n}$. Чтобы лучше понять это тонкое соотношение между интегрируемостью в размерностях 1 и 2 , рассмотрим типичный случай $Z_{m n}=-\varphi \delta_{m n}, \varphi=e^{\phi}$. Тогда уравнения (11) означают, что (заметим, что здесь $\bar{a}_{n}-1=a_{n}$ )

$$
\begin{aligned}
V(\varphi, \psi) & \equiv \phi^{\prime}(\varphi) \bar{V}(\ln \varphi, \psi)=\sum_{n=1}^{N} g_{n} \varphi^{\bar{a}_{n}-1} \exp \left[\sum_{m=3}^{N} \psi_{m} a_{m n}\right] \equiv \\
& \equiv \sum_{n=1}^{N} g_{n} \varphi^{\bar{a}_{n}-1} e^{-\bar{a}_{n} \varphi} \exp \left[\bar{a}_{n} \varphi+\sum_{m=3}^{N} \psi_{m} a_{m n}\right],
\end{aligned}
$$

где $\bar{a}_{n}, a_{m n}$ удовлетворяют условиям ортогональности (12).

Хотя теория (1) с $Z_{m n}=-\varphi \delta_{m n}$ и с потенциалом (13) заведомо неинтегрируема, в достаточно узком интервале значений $\varphi$ можно попытаться приблизить ее интегрируемой теорией ${ }^{3)}$. Во-первых, необходимо в этом интервале записать приближенное равенство $Z_{m n} \approx-\varphi_{0} \delta_{m n}$, где $\varphi_{0}>0$. Во-вторых, надо либо произвести масштабное преобразование полей материи с целью добиться того, чтобы $Z_{m n} \approx-\delta_{m n}$, либо просто поделить все члены в лагранжиане (1) на $\varphi_{0}$, а затем взять $\bar{\varphi} \equiv \varphi / \varphi_{0}$ в качестве нового дилатонного поля. Вторая идея представляется более привлекательной, так как при этом сохраняются условия псевдоортогональности для $\bar{a}_{n}, a_{m n}$, и масштабно преобразуются только константы связи $g_{n}$. После этих простых преобразований можно забыть о масштабировании и просто зафиксировать шкалу, положив $\varphi_{0}=1$. Теперь, чтобы получить интегрируемую двумерную теорию, предположим, что величины $\bar{g}_{n}(\varphi) \equiv g_{n} \varphi^{\bar{a}_{n}-1} e^{-\bar{a}_{n} \varphi}$ можно приблизить константами в некотором интервале переменной $\varphi$ вблизи $\varphi_{0}$. В простейшем приближении можно в этом выражении просто заменить $\varphi$ на $\varphi_{0}$. Более тонкое приближение можно получить, если некоторые из параметров $\bar{a}_{n}$ совпадают, например, $\bar{a}_{n}=\bar{a}$ при $n \leqslant n_{0}$. Если $g_{n}=0$ при $n_{0}<n \leqslant N$, и тем самым потенциал содержит $n_{0}$ членов, то для такого потенциала можно использовать более тонкое приближение. Производная $g_{n} \varphi^{\bar{a}_{n}-1} e^{-\bar{a}_{n} \varphi}$ равна нулю, если $\varphi=\varphi_{1} \equiv\left(\bar{a}_{n}-1\right) / \bar{a}_{n}$. Из этого следует, что если только величина $\left|\varphi-\varphi_{1}\right|$ не слишком велика, то приближение, в котором $\bar{g}_{n}(\varphi)$ заменяется на $\bar{g}_{n}\left(\varphi_{1}\right)$, оказывается оправданным при $n \leqslant n_{0}$, таким образом,

$$
V(\varphi, \psi)=\sum_{n=1}^{n_{0}} \bar{g}_{n}(\varphi) \exp \left[\bar{a} \varphi+\sum_{m=3}^{N} \psi_{m} a_{m n}\right] \approx \sum_{n=1}^{n_{0}} \bar{g}_{n}\left(\varphi_{1}\right) \exp \left[\bar{a} \varphi+\sum_{m=3}^{N} \psi_{m} a_{m n}\right] .
$$

Простейшие примеры одномерных интегрируемых $N$-лиувиллевых моделей, которые могут быть получены с помощью размерных редукций из теорий старших размерностей, имеют следующие параметры: $\bar{a}_{1}=\bar{a}, \bar{a}_{n}=-\bar{a}, n>1$, и $\bar{a}_{1}=\bar{a}_{2}=\bar{a}$,

3) Такое приближение можно было бы использовать при рассмотрении зависящих от времени явлений вблизи статических горизонтов черных дыр или при выводе приближенного описания непертурбативных короткоживущих неоднородностей в космологии. 
$\bar{a}_{n}=-\bar{a}, n>2$. Их можно получить из моделей, подробно рассмотренных в работе [25] (относительно более общих моделей см. также [5] и приложение к настоящей работе). Чтобы лучше понять вышеприведенное рассуждение, полезно рассмотреть модель из работы [25], которая представлена здесь в наших обозначениях и без привлечения рассуждений по поводу старших размерностей. Двумерный лагранжиан можно представить в виде (1) с $N=4, Z_{m n}=-\varphi \delta_{m n}$ и с потенциалом (13), в котором $\bar{a}_{n}, a_{m n}$ удовлетворяют условию (12). Ненулевые параметры модели имеют вид

$$
\bar{a}_{1}=\bar{a}_{2}=-\bar{a}_{3}=-\bar{a}_{4}=1 ; \quad a_{31}=2 \sqrt{3}, \quad a_{32}=\frac{2}{\sqrt{3}}, \quad a_{43}=-a_{44}=2 .
$$

Члены потенциала имеют следующее происхождение: первый член представляет собой кривизну трехмерной сферы, $g_{1}=6$ (этот член исчезает в пределе плоского пространства), второй и четвертый члены происходят из редукций 3-формы в лагранжиане десятимерного пространства, третий член задается абелевым калибровочным полем, порождаемым редукцией Калуцы-Клейна (KK) шестимерного пространства-времени к пятимерному; $\psi_{4}$ представляет собой скалярное поле КK, а $\psi_{3}$ - масштабный множитель пятимерной сферической редукции. Константы связи $g_{2}, g_{3}, g_{4}$ отрицательны (заметим, что $g_{n} / \gamma_{n}>0, n=1, \ldots, 4$ ). Теперь можно записать одномерный лагранжиан (8) и преобразовать его к $N$-лиувиллевому виду (10). Описанную специальную модель и ее обобщения можно использовать как “лабораторию" для сопоставления точных и приближенных решений.

Таким образом, видно, что в реалистичном случае, когда $Z_{m n}=-\varphi \delta_{m n}$, одномерную теорию можно свести к $N$-лиувиллевой модели, в то время как двумерная теория, из которой одномерная была получена с помощью наивной размерной редукции, оказывается неинтегрируемой. Хотя нам известно, что одномерные решения должны одновременно быть решениями двумерных уравнений, процедура поиска соответствующих двумерных функций требует осторожности, так как мы использовали преобразования множителей Лагранжа (в этом смысле редукция оказывается вовсе не “наивной”!). Более того, нам неизвестен полный набор решений двумерной теории, а потому мы не можем произвести непосредственную размерную редукцию на уровне решений (что можно сделать в интегрируемом случае).

Имея в виду то, что “эффективно одномерные решения”, формально зависящие от двух переменных, достаточно трудно находить в неинтегрирумой теории, проведем сначала тщательное исследование интегрируемой модели

$$
\mathcal{L}^{(2)}=\sqrt{-g}\left\{\varphi R+V(\varphi, \psi)-\sum_{n=3}^{N}\left(\nabla \psi_{n}\right)^{2}\right\}
$$

с потенциалом $V(\varphi, \psi)$, заданным формулами (3)-(9), и рассмотрим размерную редукцию ее решений к статическим состояниям, космологиям и волнам. В силу взаимосвязи между одномерной и двумерной теориями в "реалистичных" случаях 
можно надеяться, что аналогичные соображения позволят найти соответствующие редукции в реалистичных теориях с помощью процедуры обобщенной размерной редукции (в разделе 5 приводится простой пример, который указывает на такую возможность). Чтобы сделать это наблюдение более весомым, мы покажем также, как можно получить волноподобные решения $N$-лиувиллевой теории (1)-(4) с помощью процедуры обобщенного разделения переменных в уравнениях движения без построения двумерных решений общего вида.

\section{3. ОБЩЕЕ РЕШЕНИЕ $N$-ЛИУВИЛЛЕВОЙ МОДЕЛИ}

В работах [13]-[17] показано, что уравнения движения $N$-лиувиллевой модели, задаваемой уравнениями (1)-(4), значительно упрощаются, если выписать их в координатах CK (7) с использованием следующих новых функций:

$$
q_{n}(u, v) \equiv F(u, v)+q_{n}^{(0)}(u, v)=\sum_{m=1}^{N} \psi_{m} a_{m n},
$$

где $\psi_{1} \equiv(F+\varphi) / 2, \psi_{2} \equiv(F-\varphi) / 2$ и $a_{m n}$ - координаты $N$-векторов $A_{n}$, определенных выше. Применяя (5), (6), можно легко обратить (17) и выразить $\psi_{n}$ в терминах $q_{n}$ (см. [19], [16]):

$$
\psi_{n}=\sum_{m=1}^{N} \epsilon_{n} a_{n m} \gamma_{m} q_{m}, \quad \epsilon_{1}=-1, \quad \epsilon_{n}=1, \quad n>1 .
$$

Варьируя лагранжиан (16) по полям $\psi_{n}, \varphi, g_{i j}$ и преобразуя затем полученные уравнения к координатам СK, найдем $N$ уравнений движения для $\psi_{n}, n \geqslant 1$, и две связи. Выразив $\psi_{n}$ в терминах $q_{n}$, найдем тем самым $N$ уравнений Лиувилля ${ }^{4}$

$$
\partial_{u} \partial_{v} q_{n}=\tilde{g}_{n} e^{q_{n}}, \quad \tilde{g}_{n} \equiv \frac{\varepsilon g_{n}}{\gamma_{n}},
$$

при этом две связи на $N$ функций $q_{n}$ имеют вид

$$
\sum_{n=1}^{N} \gamma_{n}\left[\left(\partial_{i} q_{n}\right)^{2}-2 \partial_{i}^{2} q_{n}\right]=0, \quad i=u, v .
$$

Решение системы уравнений и связей находить легче, если определить новые функции (подсказанные конформными свойствами уравнения Лиувилля)

$$
X_{n}(u, v) \equiv e^{-q_{n}(u, v) / 2} .
$$

Тогда уравнения Лиувилля (19) можно переписать в эквивалентной форме

$$
X_{n} \partial_{u} \partial_{v} X_{n}-\partial_{u} X_{n} \partial_{v} X_{n}=-\frac{1}{2} \tilde{g}_{n}
$$

\footnotetext{
4)В физически осмысленных моделях, например, в моделях, полученных из теорий высших размерностей с помощью размерных редукций, знаки $g_{n}$ и $\gamma_{n}$ часто оказываются коррелированы так, что знаки при ненулевых константах связи $\tilde{g}_{n}$ совпадают при всех $n$.
} 
их решение должно удовлетворять уравнениям связи

$$
\sum_{n=1}^{N} \gamma_{n} X_{n}^{-1} \partial_{i}^{2} X_{n}=0, \quad i=u, v .
$$

Решение этих уравнений описано в деталях в работах [13]-[17], так что здесь мы его не повторяем. Мы только запишем более общий вид решений, который потребуется при корректной размерной редукции ${ }^{5}$.

Перейдем теперь к поиску решений общего вида для $N$-лиувиллевой теории. Дифференцируя уравнение (22) по $u$ и $v$, получим

$$
\partial_{u}\left(X_{n}^{-1} \partial_{v}^{2} X_{n}\right)=0, \quad \partial_{v}\left(X_{n}^{-1} \partial_{u}^{2} X_{n}\right)=0 .
$$

Если $X_{n}$ удовлетворяет уравнениям $(24)$, то найдутся некоторые “потенциалы” $\mathcal{U}_{n}(u)$ и $\mathcal{V}_{n}(v)$ такие, что

$$
\partial_{u}^{2} X_{n}-\mathcal{U}_{n}(u) X_{n}=0, \quad \partial_{v}^{2} X_{n}-\mathcal{V}_{n}(v) X_{n}=0
$$

Это приводит к идее ввести два обыкновенных дифференциальных уравнения

$$
a_{n}^{\prime \prime}(u)-\mathcal{U}_{n}(u) a_{n}(u)=0, \quad b_{n}^{\prime \prime}(v)-\mathcal{V}_{n}(v) b_{n}(v)=0 .
$$

Тогда решения $X_{n}$ уравнений Лиувилля можно выразить через решения $a_{n}(u)$ и $b_{n}(v)$ этих уравнений, которые можно выбрать произвольными; при этом линейно независимые решения могут быть найдены, даже если неизвестен конкретный вид потенциала ${ }^{6)}$. Тем не менее все это не помогает в разрешении связей $(23)$, которые, как мы покажем ниже, принимают вид

$$
\sum_{n=1}^{N} \gamma_{n} \frac{a_{n}^{\prime \prime}(u)}{a_{n}(u)}=0, \quad \sum_{n=1}^{N} \gamma_{n} \frac{b_{n}^{\prime \prime}(v)}{b_{n}(v)}=0
$$

где $a_{n}(u)$ и $b_{n}(v)$ - произвольные решения уравнений (27). Хотя можно выбрать произвольными $2(N-1)$ потенциалов из $\mathcal{U}_{n}(u), \mathcal{V}_{n}(v)$ и соответствующие $a_{n}(u), b_{n}(v)$, две оставшихся функции $a, b$ удовлетворяют уравнениям второго порядка с произвольными потенциалами. Эти уравнения не удается решить, если $\gamma_{n}$ - произвольные числа. В работах [13]-[17] мы показали, что можно построить решение, используя то обстоятельство, что в $N$-лиувиллевой теории $\sum_{n=1}^{N} \gamma_{n}$ обращается в нуль. Этот результат несколько обобщается ниже ${ }^{7)}$.

5) Решения, использованные в предшествующих работах, были по существу правильными, но они были записаны в вводящем в некоторое заблуждение виде, который мог спровоцировать их неправильное использование; ниже это станет очевидным.

6)Этот вид общего решения уравнения Лиувилля был отмечен в работе [26]. Его теоретико-групповой смысл и приложения обсуждались в работах [27], [28], где можно найти также необходимые ссылки.

7) Для некоторых специальных потенциалов уравнения (26) удается разрешить даже при произвольных $\gamma_{n}$. Ниже подробно исследуется случай постоянных потенциалов, который можно рассматривать как некоторую обобщенную размерную редукцию $N$-лиувиллевой теории в присутствии или отсутствии связей. 
Вводя линейно независимые решения $a_{n}^{(1)}(u), a_{n}^{(2)}(u)$ и $b_{n}^{(1)}(v), b_{n}^{(2)}(v)$ уравнений (26), нормированные условием равенства единице вронскианов,

$$
W\left[a_{n}^{(1)}(u), a_{n}^{(2)}(u)\right]=1, \quad W\left[b_{n}^{(1)}(v), b_{n}^{(2)}(v)\right]=1,
$$

можно показать, что $X_{n}$, удовлетворяющие уравнению $(24)$, должны иметь вид

$$
X_{n}(u, v)=a_{n}^{(i)}(u) C_{i j}^{(n)} b_{n}^{(j)}(v)
$$

где $C_{i j}^{(n)}$ - числовая матрица (и производится суммирование по $\left.i, j=1,2\right)$. Нетрудно проверить, что $X_{n}$ удовлетворяет уравнениям (22) тогда и только тогда, когда

$$
\operatorname{det} C_{i j}^{(n)}=-\frac{\tilde{g}_{n}}{2}
$$

В работах [13]-[16] была использована упрощенная форма решения, полученная при $C_{12}^{(n)}=C_{21}^{(n)}=0, C_{11}^{(n)}=1$ и $C_{22}^{(n)}=-\tilde{g}_{n} / 2$. Общее решение можно получить из упрощенного, если применить линейные преобразования базисных киральных полей $a_{n}^{(i)}(u), b_{n}^{(j)}(v)$, которые сохраняют вронскианы:

$$
a_{n}^{(i)}(u) \rightarrow A_{i j}^{(n)} a_{n}^{(j)}(u), \quad b_{n}^{(i)}(v) \rightarrow B_{i j}^{(n)} b_{n}^{(j)}(v),
$$

где $\operatorname{det} A_{i j}^{(n)}=\operatorname{det} B_{i j}^{(n)}=1$. Хотя упрощенное представление решения можно с соблюдением осторожности использовать в общих построениях, общее решение (29) оказывается более адекватным для анализа физических свойств решений и особенно важным при дальнейших размерных редукциях, как будет показано ниже.

Как и в предыдущих работах, можно положить $a_{n}^{(1)}(u) \equiv a_{n}(u)$ и $b_{n}^{(1)}(v) \equiv b_{n}(v)$, где $a_{n}(u)$ и $b_{n}(v)$ - произвольные функции, и взять

$$
a_{n}^{(2)}(u) \equiv \bar{a}_{n}(u) \equiv a_{n}(u) \int \frac{d u}{a_{n}^{2}(u)}, \quad b_{n}^{(2)}(v) \equiv \bar{b}_{n}(v) \equiv b_{n}(v) \int \frac{d v}{b_{n}^{2}(v)} .
$$

Это позволит применить рассуждения предыдущих статей к общим решениям $X_{n}$. Ключевой факт, с помощью которого стало возможным разрешить связи в работах [13]-[16], состоит в том, что эти связи эквивалентны связям (27), и из условий псевдоортогональности следует тождество

$$
\sum_{n=1}^{N} \gamma_{n}=0
$$

В самом деле, имеем

$$
\frac{\partial_{u}^{2} X_{n}}{X_{n}}=\frac{\left[\partial_{u}^{2} a_{n}^{(i)}(u)\right] C_{i j}^{(n)} b_{n}^{(i)}(v)}{a_{n}^{(i)}(u) C_{n}^{(i j)} b_{n}^{(j)}(v)}=\mathcal{U}_{n}(u)=\frac{a_{n}^{\prime \prime}(u)}{a_{n}(u)}=\frac{\bar{a}_{n}^{\prime \prime}(u)}{\bar{a}_{n}(u)},
$$

6 Теоретическая и математическая физика, т. 153, № 3, 2007 г. 
аналогичное выражение справедливо для функций $b_{n}(v), \bar{b}_{n}(v)$. Отсюда следует, что связи (23) имеют вид (27), и это позволило разрешить их (см. работы [13]-[16]). Применяя теперь конструкцию из этих работ, мы можем записать решение уравнений и связей с теми же выражениями для базисных функций $a_{n}$ и $b_{n}$ :

$$
\begin{aligned}
& a_{n}(u)=\left|\sum \gamma_{m} \mu_{m}(u)\right|^{-1 / 2} \exp \left\{\int d u \mu_{n}(u)\right\}, \\
& b_{n}(u)=\left|\sum \gamma_{m} \nu_{m}(v)\right|^{-1 / 2} \exp \left\{\int d u \nu_{n}(v)\right\},
\end{aligned}
$$

где функции $\mu_{n}(u), \nu_{n}(v)$, называемые модулями, удовлетворяют связям ${ }^{8)}$

$$
\sum_{n=1}^{N} \gamma_{n} \mu_{n}^{2}(u)=0, \quad \sum_{n=1}^{N} \gamma_{n} \nu_{n}^{2}(v)=0 .
$$

Подставляя (34), (35) в (29), найдем $q_{n}$, после чего, используя условия ортогональности, можно записать исходные поля $\psi_{n}, \phi, F$ в терминах $a_{n}, b_{n}$ (см. [13]-[16]; соответствующие выражения воспроизведены в следующем разделе).

Как было показано в работах [14], [16], пространство модулей $\left(\mu_{1}, \ldots, \mu_{n}\right)$, $\left(\nu_{1}, \ldots, \nu_{n}\right)$ можно редуцировать дальше, если наложить два координатных условия, фиксирующих калибровку. В работах [14], [16] было предложено ввести новые координаты $(U, V)$, положив

$$
\sum \gamma_{n} \mu_{n}(u)=U^{\prime}(u), \quad \sum \gamma_{n} \nu_{n}(v)=V^{\prime}(v),
$$

но в некоторых задачах другой выбор координатных условий может оказаться более удобным.

В отмеченных работах было показано, что из условий псевдоортогональности следует, что все $\gamma_{n}$, кроме одного, должны быть положительны, так что всегда выбирается $\gamma_{1}<0$. Отсюда следует, что в силу условий связи функции $\mu_{1}(u)$ и $\nu_{1}(v)$ не могут обращаться в нуль ${ }^{9)}$. Следовательно,

$$
\int \mu_{1}(u) d u, \quad \int \nu_{1}(v) d v
$$

- монотонные функции, и их поэтому можно выбрать в качестве новых координат, т.е. положить

$$
U \equiv \int \mu_{1}(u) d u, \quad V \equiv \int \nu_{1}(v) d v .
$$

8) Потенциалы $\mathcal{U}_{n}(u), \mathcal{V}_{n}(v)$ легко записываются в терминах модулей, но эти сложные формулы не позволяют найти обратные выражения, за исключением случая постоянных модулей, рассмотренного в следующем разделе.

9) Это не так, если все $\mu_{n}$ или $\nu_{n}$ обращаются в нуль. Тогда связи удовлетворяются тождественно, но этот чрезвычайно вырожденный случай не представляет интереса. Более интересным оказывается случай мнимых модулей, к которому наш подход применим в полной мере. Мы не рассматриваем соответствующие решения, поскольку в них сингулярности могут возникать при конечных значениях $u$ и $v$. В данной работе основной упор сделан на решения, которые могут иметь сингулярность в пространстве $(u, v)$ только в бесконечно удаленной точке. 
Это, очевидно, эквивалентно выбору $\mu_{1}(u)$ и $\nu_{1}(v)$ в виде

$$
\mu_{1}(u) \equiv \nu_{1}(v) \equiv 1
$$

при использовании исходных координат $(u, v)$.

Простой способ объединить все вышеуказанные свойства пространства модулей состоит в том, чтобы ввести единичные векторы (см. [14], [16])

$$
\hat{\xi}_{k}(u) \equiv \hat{\gamma}_{k} \mu_{k}(u), \quad \hat{\eta}_{k}(v) \equiv \hat{\gamma}_{k} \nu_{k}(v), \quad k=2,3, \ldots, N
$$

где $\hat{\gamma}_{k} \equiv \sqrt{\gamma_{k} /\left|\gamma_{1}\right|}$. Из известных свойств параметров $\gamma_{k}$ и условий связи на переменные $\mu_{n}$ и $\nu_{n}$ (включая $\mu_{1}=\nu_{1}=1$ ) видно, что

$$
\hat{\xi}^{2} \equiv \sum_{k=2}^{N} \hat{\xi}_{k}^{2}=1, \quad \hat{\eta}^{2} \equiv \sum_{k=2}^{N} \hat{\eta}_{k}^{2}=1, \quad \hat{\gamma}^{2} \equiv \sum_{k=2}^{N} \hat{\gamma}_{k}^{2}=1
$$

Независимые модули $\hat{\xi}(u), \hat{\eta}(v)$ лежат на сфере $S^{(N-2)}$, и пространство модулей состоит из пар непрерывных траекторий $(\hat{\xi}(u), \hat{\eta}(v))$ на $S^{(N-2)}$. Пары точек $\left(\hat{\xi}^{(0)}, \hat{\eta}^{(0)}\right)$ также задают интересный класс решений, который детально исследован ниже $^{10)}$. Модули $\hat{\xi}(u)$ и $\hat{\eta}(v)$ должны быть определены для всех вещественных значений координат $(u, v)$. На сфере $S^{(N-2)}$ эти траектории могут быть замкнутыми кривыми, быть бесконечными в обоих направлениях или даже иметь одну или две конечных концевых точки. Если, например, траектория $\hat{\xi}(u)$ имеет концевую точку $\hat{\xi}^{(0)}$, то это означает, что $\hat{\xi}(u) \rightarrow \hat{\xi}^{(0)}$ при $u \rightarrow+\infty$ (аналогично точка $\hat{\xi}^{(0)}$ может служить начальной точкой траектории, если $\xi(u) \rightarrow \hat{\xi}^{(0)}$ при $\left.u \rightarrow-\infty\right)$.

Применяя эти простые свойства, можно попытаться построить классификацию траекторий, основанную на их топологических и асимптотических свойствах. Это вполне очевидно для $S^{(1)}$ и достаточно просто для $S^{(2)}$. В старших размерностях (начиная с $S^{(3)}$ ) может оказаться, что найти какую-либо классификацию для всех возможных траекторий весьма непросто. Во всех размерностях, однако, существует класс физически значимых решений, задаваемых точками $\left(\hat{\xi}^{(0)}, \hat{\eta}^{(0)}\right)$. Эти решения в действительности определяют некоторые нетривиальные размерные редукции $(1+1)$-мерных решений и могут описывать статические черные дыры (или, в более общем понимании, статические состояния гравитирующей материи), космологии и различные типы нелинейных волн, взаимодействующих с гравитацией. Среди этих волновых решений могут присутствовать волны, конечные всюду в пространстве и времени, включая бесконечность. Все эти решения будут наиболее важными для дальнейшего обсуждения.

10) В наших предыдущих работах в основном исследовались решения, для которых $\hat{\xi}^{(0)}=\hat{\eta}^{(0)}$. Статические решения с двумя горизонтами описываются решениями, задаваемыми условиями $\hat{\xi}^{(0)}=\hat{\eta}^{(0)}=\hat{\gamma}$ (см. работу [13]). Они представляют собой весьма специальные примеры решений, обсуждаемых в следующем разделе. 
Приведем сначала несколько замечаний относительно размерной редукции как продолжение обсуждения этого предмета, начатого в предшествующих работах [14], [16]. При "наивной" редукции просто уменьшается размерность пространства-времени, на котором определены динамические функции (например, $q_{n}(u, v)$ ), с помощью построения подкласса решений, зависящих только от одной переменной (например, $\left.q_{n}(t) \equiv q_{n}(u-v)\right)$. Для функций $(1+1)$-мерной дилатонной теории гравитации эта наивная редукция детально обсуждалась в работе [14]. Там же было указано, что наивная редукция к переменным, зависящим только от времени, не воспроизводит всех возможных космологий. Имеются также указания на то, что редукция старших размерностей $D$ к размерности $1+0$ не эквивалентна редукции размерности $1+1$ к 1+0. В приложении 6.2 работы [14] было показано, как воспроизвести все возможные однородные и изотропные космологии, рассмотрев более общую редукцию $(1+1)$-мерной теории.

В более общих подходах к редукциям используются теоретико-групповые соображения (см., например, [29]-[33] и ссылки в этих работах), но их не всегда легко непосредственно применить к редукции уравнений движения. Процедура обобщенного разделения переменных, введенная в работе [17], задает еще более общий подход к размерной редукции. В частности, будучи примененным к теориям в размерности $1+1$, этот подход позволяет воспроизвести все известные решения типа черных дыр и космологий [17]. Кроме того, он оказывается хорошо применимым к случаю, когда дилатонная гравитация взаимодействует с произвольным числом полей материи, и с его помощью получаются (вероятно) новые решения, которые, будучи по существу одномерными, зависят от обеих переменных $r$ и $t$.

В настоящей работе мы исследуем размерную редукцию в интегрируемой $N$-лиувиллевой теории, дающую класс редуцированных решений, которые зависят от комбинаций обеих переменных. Пример решений такого класса приводится в конце раздела 3 работы [16]; он был получен с помощью размерной редукции пространства модулей. Идею такой размерной редукции (временно будем ее называть “динамической размерной редукцией”) легко сформулировать в терминах описанного выше пространства модулей. В то время как решения в $(1+1)$-мерной теории задаются парами функций $(\hat{\xi}(u), \hat{\eta}(v))$, редуцированные решения параметризуются точками $\left(\hat{\xi}^{(0)}, \hat{\eta}^{(0)}\right)$. Эти решения описывают волны скалярной материи, и среди их специальных случаев встречаются статические состояния (в частности, черные дыры) и космологии. Таким образом, все эти объекты объединяются в один более общий класс.

Решения будут исследованы в следующем разделе, где мы попробуем выделить те из них, в которых поля материи остаются конечными как при $r \rightarrow \infty$, так и при $t \rightarrow \infty$. Затем мы покажем, что те же самые состояния можно построить, используя вместо динамической редукции процедуру обобщенного разделения переменных пространства и времени. Это довольно простое упражнение, не требующее знания 
$(1+1)$-мерных решений. Поэтому его можно применить и к неинтегрируемым задачам. Этот факт обсуждается в конце статьи на очень простом примере.

\section{4. СОСТОЯнИЯ С ПОстОЯнНЫМИ МОДУЛЯМИ}

Рассмотрим решения с постоянными модулями $\mu_{n}, \nu_{n}$. Тогда $\mathcal{U}_{n}(u)=\mu_{n}^{2}$, $\mathcal{V}_{n}(u)=\nu_{n}^{2}$, и мы можем легко выписать явно базисные киральные поля. Если ни один из модулей не равен нулю ${ }^{11)}$, то в соответствии с условиями (26) можно использовать следующий набор:

$$
\begin{aligned}
a_{n}(u) & =\frac{1}{\sqrt{2 \mu_{n}}} e^{-\mu_{n} u}, & b_{n}(u) & =\frac{1}{\sqrt{2 \nu_{n}}} e^{-\nu_{n} u}, \\
\bar{a}_{n}(u) & =\frac{1}{\sqrt{2 \mu_{n}}} e^{\mu_{n} u}, & \bar{b}_{n}(u) & =\frac{1}{\sqrt{2 \nu_{n}}} e^{\nu_{n} u} .
\end{aligned}
$$

Теперь общие решения уравнений (22) с постоянными модулями $\mu_{n}$ и $\nu_{n}$ можно записать как

$X_{n}(u, v)=\frac{1}{2 \sqrt{\mu_{n} \nu_{n}}}\left[C_{11}^{(n)} e^{-\mu_{n} u-\nu_{n} v}+C_{22}^{(n)} e^{\mu_{n} u+\nu_{n} v}+C_{12}^{(n)} e^{-\mu_{n} u+\nu_{n} v}+C_{21}^{(n)} e^{\mu_{n} u-\nu_{n} v}\right]$,

где

$$
C_{11}^{(n)} C_{22}^{(n)}-C_{12}^{(n)} C_{21}^{(n)}=-\frac{1}{2} \tilde{g}_{n}
$$

В общем случае, когда все элементы $C_{i j}^{(n)} \neq 0$, можно без ограничения общности считать все модули неотрицательными, $\mu_{n} \geqslant 0, \nu_{n} \geqslant 0$. Если не все из них равны нулю, то $\mu_{1} \neq 0, \nu_{1} \neq 0$ ввиду условий связи (напомним, что $\gamma_{1}<0$ и $\gamma_{k}>0$ )

$$
\left|\gamma_{1}\right| \mu_{1}^{2}=\sum_{k=2}^{N} \gamma_{k} \mu_{k}^{2}, \quad\left|\gamma_{1}\right| \nu_{1}^{2}=\sum_{k=2}^{N} \gamma_{k} \nu_{k}^{2}
$$

Чтобы упростить изложение, предположим в дальнейшем, что $\mu_{n}>0, \nu_{n}>0$. Напомним также, что можно выбрать калибровку, в которой $\mu_{1}=\nu_{1}=1$. В этой калибровке имеем $\mu_{k} \leqslant \sqrt{\left|\gamma_{1}\right| / \gamma_{k}}, k>1$.

Знаки матричных элементов $C_{i j}^{(n)}$ могут быть, вообще говоря, различными, а потому $X_{n}(u, v)$ могут иметь нули в некоторых конечных точках ${ }^{12)}, X_{n}\left(u_{0}, v_{0}\right)=0$ и соответственно $q_{n}\left(u_{0}, v_{0}\right)=-2 \ln \left|X_{n}\left(u_{0}, v_{0}\right)\right|=\infty$. В общем случае поля материи также могут обращаться в бесконечность в этих точках. Чтобы избежать появления этих сингулярностей, предположим, что все $C_{i j}^{(n)}$ положительны ${ }^{13)}$. Разумеется,

11) Если позволить каким-то из $\mu_{n}$ обращаться в нуль, можно использовать другие базисные функции, например $a_{n}=\operatorname{ch} \mu_{n}, \bar{a}_{n}=\mu_{n}^{-1} \operatorname{sh} \mu_{n}$, но эти тонкости остаются за рамками нашего рассмотрения.

12) Будем в дальнейшем, как правило, предполагать, что $-\infty<u<+\infty,-\infty<v<+\infty$.

13) Наличие сингулярностей представляет собой характерное свойство уравнения Лиувилля, которое было тщательно изучено различными исследователями в прошлом (см., например, работы [26], [34], [35]). Как объясняется во введении, наша цель состоит в исследовании решений с минимально возможными сингулярностями, а потому здесь разрешены лишь сингулярности на бесконечности. 
специальные решения, в которых $C_{12}^{(n)}=C_{21}^{(n)}=0$ или $C_{11}^{(n)}=C_{22}^{(n)}=0$ при всех $n$ также представляют интерес, поскольку, как будет очень скоро ясно, они могут описывать статические или космологические состояния. Заметим, что наши замечания относительно сингулярностей в полной мере применимы к общему решению (29). Чтобы избежать появления сингулярностей в конечных точках, необходимо предположить, что базисные киральные функции положительны, а матричные элементы $C_{i j}^{(n)} \geqslant 0$ (некоторые из них должны быть ненулевыми).

В случае положительных $C_{i j}^{(n)}$ представляется естественным переписать решения в виде

$$
X_{n}(u, v)=\frac{1}{\sqrt{\mu_{n} \nu_{n}}}\left\{C_{n}^{+} \operatorname{ch}\left(\mu_{n} u+\nu_{n} v+\delta_{n}^{+}\right)+C_{n}^{-} \operatorname{ch}\left(\mu_{n} u-\nu_{n} v+\delta_{n}^{-}\right)\right\},
$$

где $C_{n}^{ \pm}, \delta_{n}^{ \pm}$задаются уравнениями

$$
\begin{aligned}
C_{n}^{+}=C_{11}^{(n)} e^{\delta_{n}^{+}} & =C_{22}^{(n)} e^{-\delta_{n}^{+}}, & C_{n}^{-} & =C_{12}^{(n)} e^{\delta_{n}^{-}}=C_{21}^{(n)} e^{-\delta_{n}^{-}}, \\
\delta_{n}^{+} & =\frac{1}{2} \ln \frac{C_{11}^{(n)}}{C_{22}^{(n)}}, & \delta_{n}^{-} & =\frac{1}{2} \ln \frac{C_{12}^{(n)}}{C_{21}^{(n)}}
\end{aligned}
$$

в силу (46) отсюда получим, что

$$
\left(C_{n}^{+}\right)^{2}-\left(C_{n}^{-}\right)^{2}=-\frac{\tilde{g}_{n}}{2}
$$

Отметим важное свойство этой простой формулы: равенство $C_{n}^{-}=0$ возможно, только если $\tilde{g}_{n}<0$, а равенство $C_{n}^{+}=0$ - только при $\tilde{g}_{n}>0$. Поскольку $\tilde{g}_{n} \equiv \varepsilon g_{n} / \gamma_{n}$, где $\varepsilon$ - знак метрики $f$, мы приходим к следующему наблюдению: так как $\gamma_{1}<0$ и $\gamma_{k}>0$ при $k>1$, случай, в котором все $\tilde{g}_{n}$ имеют один и тот же знак, возможен, только если $g_{1} / g_{k}<0$ (или $g_{1}=0$ ). Этот факт оказывается важным при построении стандартных одномерных решений.

Статические или космологические решения можно получить из (48), положив $\mu_{n}=\nu_{n}$ и $C_{n}^{-}=0$ или $C_{n}^{+}=0$ при всех $n$. Тогда статические состояния зависят от пространственных координат, заданных как $r \equiv(u-\varepsilon v)$, в то время как космологические состояния зависят от временно́й координаты $t \equiv(u+\varepsilon v)^{14)}$. Заметим, что для построения из (45) всех возможных статических и космологических решений, необходимо было бы допустить не только положительные матричные элементы $C_{i j}^{(n)}$. Заметим также, что в общем случае можно взять мнимые $\mu_{n}$ и $\nu_{n}$, сохраняя $X_{n}(u, v)$ вещественными, но в этой работе мы не рассматриваем осциллирующих волн, которые обязательно имеют сингулярности в конечных точках пространства $(u, v)$.

14) Разумеется, идентифицировать по-настоящему пространственную и временну́ю координаты в размерности $1+1$ можно, только если связать двумерную метрику с метрикой старших размерностей (см. обсуждение в работе [14]). В дальнейшем мы обычно полагаем $r=u+v$ и $t=u-v$, принимая в расчет, что все формулы по существу симметричны относительно замены $r \leftrightarrow t$. 
Для того чтобы добиться лучшего понимания физического смысла решений, перепишем их как функции от $r$ и $t$ :

$$
X_{n}=\frac{1}{\sqrt{\mu_{n} \nu_{n}}}\left\{C_{n}^{+} \operatorname{ch}\left(\lambda_{n} r+\bar{\lambda}_{n} t+\delta_{n}^{+}\right)+C_{n}^{-} \operatorname{ch}\left(\lambda_{n} t+\bar{\lambda}_{n} r+\delta_{n}^{-}\right)\right\},
$$

где использованы обозначения $\lambda_{n}=\left(\mu_{n}+\nu_{n}\right) / 2$ и $\bar{\lambda}_{n}=\left(\mu_{n}-\nu_{n}\right) / 2$. Видно, что $X_{n}$ представляют собой суперпозиции двух волн с фазовыми скоростям $V_{n}^{-} \equiv \lambda_{n} / \bar{\lambda}_{n}$ и $V_{n}^{+} \equiv \bar{\lambda}_{n} / \lambda_{n}$

Обратимся теперь к исследованию асимптотического поведения полей $\psi_{m}, \varphi, F$ в случае решений вида (48). Сначала выпишем и обсудим вкратце некоторые общие формулы. Как следует из уравнений (46), (47) работы [16], выражения для исходных полей в терминах $X_{n}$ имеют следующий вид:

$$
\begin{aligned}
\psi_{m} & =-2 \sum_{n=1}^{N} a_{m n} \gamma_{n} \ln \left|X_{n}(u, v)\right|, \quad m \geqslant 3, \\
\varphi & =4 \sum_{n=1}^{N} \gamma_{n} \ln \left|X_{n}(u, v)\right|, \\
F & =4 \sum_{n=1}^{N} a_{n} \gamma_{n} \ln \left|X_{n}(u, v)\right|,
\end{aligned}
$$

а кривизна $(1+1)$-мерного пространства-времени задается формулой

$$
R=e^{-F} \partial_{u} \partial_{v} F=-\prod_{n=1}^{N}\left|X_{n}(u, v)\right|^{-4 a_{n} \gamma_{n}} \cdot \sum_{m=1}^{N} 2 a_{m} \gamma_{m} \tilde{g}_{m} X_{m}^{-2},
$$

где используется уравнение (22).

Как было указано выше, мы предполагаем, что решения не имеют сингулярностей в конечных точках пространства $(u, v)$. В общем случае у них имеются сингулярности, когда переменные $(u, v)$ (или $(r, t))$ обращаются в бесконечность. Тем не менее при определенном выборе модулей бесконечности могут сокращаться. Например, если все функции $X_{n}$ бесконечны и величины $\ln \left|X_{n}(u, v)\right|$ имеют одно и то же асимптотическое поведение, т.е.

$$
\ln \left|X_{n}(u, v)\right|=\ln |X(u, v)|+f_{n}(u, v),
$$

где функции $f_{n}(u, v)$ асимптотически конечны, то $\psi_{m}$ и $\varphi$ могут быть конечными, а $F \rightarrow-\infty$ (это означает, что $|f|=e^{F} \rightarrow 0$ ). Это можно получить из соотношений (см. [14], [16])

$$
\sum_{n=1}^{N} \gamma_{n}=0 ; \quad \sum_{n=1}^{N} a_{m n} \gamma_{n}=0, \quad m \geqslant 3 ; \quad 4 \sum_{n=1}^{N} a_{n} \gamma_{n}=-2 .
$$

Также имеем, что $R$ в данном случае конечно. На самом деле, как было показано в работах [14], [16], такое поведение можно обеспечить для размерно редуцированных 
статических (зависящих только от $r$ ) решений, имеющих горизонты при $r \rightarrow \pm \infty$, когда $|f| \rightarrow 0$.

Обратимся теперь к размерно редуцированному решению общего вида (48) с постоянными $\mu_{n}$ и $\nu_{n}$. Будем искать минимально сингулярные решения, конечные на пространственной и/или временно́й бесконечности, которые можно рассматривать как локализованные в некотором слабом смысле. Исследуя сингулярности на бесконечности решений с постоянными модулями, будем использовать общие формулы (53)-(55) для нахождения их поведения при $r \rightarrow \infty$ и/или $t \rightarrow \infty$. Нетрудно найти следующие асимптотические формулы:

$$
\begin{aligned}
& \ln \left|X_{n}(u, v)\right|_{r \rightarrow \pm \infty}= \pm\left(\lambda_{n} r+\bar{\lambda}_{n} t+\delta_{n}^{+}\right)+\ln \frac{C_{n}^{+}}{2 \sqrt{\mu_{n} \nu_{n}}}+\cdots, \\
& \ln \left|X_{n}(u, v)\right|_{t \rightarrow \pm \infty}= \pm\left(\lambda_{n} t+\bar{\lambda}_{n} r+\delta_{n}^{-}\right)+\ln \frac{C_{n}^{-}}{2 \sqrt{\mu_{n} \nu_{n}}}+\cdots
\end{aligned}
$$

Исключенные члены оказываются экспоненциально малыми в двух случаях: во-первых, если $r \rightarrow \pm \infty$ и $t$ конечно или $t \rightarrow \pm \infty$, но $|t| /|r| \leqslant 1-\epsilon$, где $\epsilon-$ произвольно малое положительное число (см. (58)); во-вторых, если $t \rightarrow \pm \infty$ и $r$ конечно или $r \rightarrow \pm \infty$, но $|r| /|t| \leqslant 1-\epsilon$ (см. уравнение (59)).

Видно, что поля обычно обращаются в бесконечность при бесконечных значениях аргументов. Однако первые производные полей конечны на бесконечности, а потому и всюду; более того, так как модули ограничены сверху, первые производные также ограничены. Вторые производные и функции $e^{q_{n}}$ локализованы в пространстве и времени, поскольку экспоненциально убывают на бесконечности.

Подводя итог, отметим что если мы рассматривамем условие конечности выражений для $\psi_{m}, \varphi, F$, то можно подставить в выражения (58), (59) лишь один расходящийся член

$$
-\frac{q_{n}^{\infty}}{2}=\ln \left|X_{n}^{\infty}\right|=\lambda_{n}|\tau|, \quad|\tau| \rightarrow \infty,
$$

где либо $\tau=r \rightarrow \pm \infty$ (и $t$ конечно), либо $\tau=t \rightarrow \pm \infty$ (и $r$ конечно). Случаи, когда $|r| \rightarrow \infty$ и $|t| \rightarrow(1-\epsilon) r$ или $|t| \rightarrow \infty$ и $|r|=|t|(1-\epsilon)$ можно рассмотреть аналогично.

Таким образом, видно, что расходящиеся части в выражениях для полей (53)-(55) и кривизны $(56)$ имеют вид

$$
\begin{aligned}
\psi_{m}^{\infty} & =-2 \sum_{n=1}^{N} a_{m n} \gamma_{n} \lambda_{n}|\tau|, \quad 3 \leqslant m \leqslant N, \\
\varphi^{\infty} & =4 \sum_{n=1}^{N} \gamma_{n} \lambda_{n}|\tau|, \\
F^{\infty} & =4 \sum_{n=1}^{N} a_{n} \gamma_{n} \lambda_{n}|\tau| \\
R^{\infty} & =-\exp \left[-2|\tau|\left(2 \sum_{n=1}^{N} a_{n} \gamma_{n} \lambda_{n}-\lambda_{\min }\right)\right],
\end{aligned}
$$


где $\lambda_{\min }-$ минимальное значение $\lambda_{n}$ при $1 \leqslant n \leqslant N$. Чтобы выражения для полей $\psi_{m}, \varphi, F$ были конечны, члены $\psi_{m}^{\infty}, \varphi^{\infty}$ и $F^{\infty}$ должны обратиться в нуль ${ }^{15)}$, при этом $R$ будет принимать значение 0 или $\infty$ в зависимости от знака выражения в круглых скобках в (64); значение $R$ будет конечным, если это выражение равно нулю. Для произвольно заданной $N$-лиувиллевой модели параметры $a_{m n}, a_{n}$ и $\gamma_{n}$ фиксированы, и можно взять как раз те параметры $\lambda_{n}$, которые удовлетворяют приведенным выше ограничениям. Конечно, не все из этих ограничений можно удовлетворить одновременно.

Предположим, что мы решили, например, уравнения $\psi_{m}^{\infty}=0$ и нашли $\lambda_{n}$. Напомним, что $\mu_{n}=\lambda_{n}+\bar{\lambda}_{n}, \nu_{n}=\lambda_{n}-\bar{\lambda}_{n}$. Отсюда можно непосредственно увидеть, что для того чтобы были выполнены условия (47), на $\lambda_{n}$ (и $\left.\bar{\lambda}_{n}\right)$ должны быть наложены некоторые ограничения. Наиболее простой способ найти эти ограничения состоит в использовании единичных векторов (41), которые в данном случае представляют собой константы. Напомним, что

$$
\hat{\xi}_{k}=\hat{\gamma}_{k}\left(\lambda_{k}+\bar{\lambda}_{k}\right), \quad \hat{\eta}_{k}=\hat{\gamma}_{k}\left(\lambda_{k}-\bar{\lambda}_{k}\right), \quad k=2, \ldots, N
$$

и зададим векторы

$$
\hat{\lambda}_{k}^{+}=\hat{\gamma}_{k} \lambda_{k}=\frac{\hat{\xi}_{k}+\hat{\eta}_{k}}{2}, \quad \hat{\lambda}_{k}^{-}=\hat{\gamma}_{k} \bar{\lambda}_{k}=\frac{\hat{\xi}_{k}-\hat{\eta}_{k}}{2}, \quad k=2, \ldots, N
$$

которые удовлетворяют двум ограничениям (условиям нормировки и ортогональности):

$$
\hat{\lambda}_{+}^{2}+\hat{\lambda}_{-}^{2}=1, \quad \hat{\lambda}_{+} \cdot \hat{\lambda}_{-}=0
$$

Легко увидеть, что эти два условия эквивалентны ограничениям (47).

Используя эти определения, можно построить решения следующим образом. Сначала выберем произвольный $(N-2)$-вектор $\hat{\lambda}_{+}$с нормой, не превосходящей 1 , а затем выберем произвольный вектор, ортогональный $\hat{\lambda}_{+}$и имеющий норму $\sqrt{1-\hat{\lambda}_{+}^{2}}$. Такая процедура задает общее решение. Однако если $\lambda_{k}$ удовлетворяют некоторым дополнительным ограничениям (например, условию конечности $\psi_{n}$ ), то обеспечение выполнения условия $\hat{\lambda}_{+}^{2} \leqslant 1$ представляет собой нетривиальную задачу. В самом деле, если $\lambda_{k}$ были получены из некоторых уравнений, то следует проверить, что

$$
\hat{\lambda}_{+}^{2} \equiv \sum_{k=2}^{N} \hat{\gamma}_{k}^{2} \lambda_{k}^{2} \leqslant 1,
$$

и мы сразу видим, что это условие действительно налагает весьма серьезные ограничения. Если это соотношение выполнено, то оставшаяся процедура уже описана

15) Условие конечности полей $\psi_{n}$ физически оправданно, но не является абсолютно необходимым. Также в общем случае мы не требуем конечности полей $\phi$ и $F$ при бесконечных аргументах. Так как первые производные всех этих полей конечны всюду, включая бесконечность, соответствующие плотности энергии и импульса конечны всюду при произвольном выборе модулей. 
выше - выбираем в качестве $\hat{\lambda}_{-}$произвольный вектор, ортогональный $\hat{\lambda}_{+}$и имеющий норму $\sqrt{1-\hat{\lambda}_{+}^{2}}$. Тогда в соответствии с нашим общим построением векторы

$$
\hat{\xi}=\hat{\lambda}_{+}+\hat{\lambda}_{-}, \quad \hat{\eta}=\hat{\lambda}_{+}-\hat{\lambda}_{-}
$$

задают решение, удовлетворяющее ограничениям (47).

Рассмотрим сначала условия на $\lambda_{n}$, налагаемые равенствами $\psi_{m}^{\infty}=\varphi^{\infty}=F^{\infty}=0$. Применяя тождества (57), найдем, что условия $\psi_{m}^{\infty}=0, \varphi^{\infty}=0$ и $F^{\infty}=0$ задают соответственно следующие уравнения на $y_{k} \equiv \gamma_{k}\left(\lambda_{k}-1\right)$ :

$$
\begin{gathered}
\sum_{k=2}^{N} a_{m k} y_{k}=0, \quad m=3, \ldots, N, \\
\sum_{k=2}^{N} y_{k}=0, \\
\sum_{k=2}^{N} a_{k} y_{k}=\frac{1}{2} .
\end{gathered}
$$

В общем случае эта система уравнений не имеет решения, так как является системой из $N$ линейных уравнений на $N-1$ параметр $y_{k}$. Однородные уравнения $(70),(71)$ всегда имеют нулевое решение $y_{k}=0$, которое дает $\mu_{n}=\nu_{n}=1$ (так как $\mu_{1}=$ $\left.\nu_{1}=1\right)$. Это отвечает статическому решению с двумя горизонтами (см. [14], [16]).

Заметим, что уравнения $(70),(71)$ могут иметь нетривиальные $\left(y_{k} \neq 0\right)$ решения в случае, когда определитель матрицы этих уравнений обращается в нуль. Конечно, это выполняется только для некоторых специальных систем, и мы не рассматриваем эту возможность в общем случае. Заметим лишь, что это невозможно при $N=3$ (единственное скалярное поле), так как в этом случае в силу $a_{33}=a_{32}$ величины $\gamma_{2}^{-1}$ и $\gamma_{3}^{-1}$ должны обращаться в нуль (см. приложение в работе [16]). Исследование случая $N=4$ гораздо более громоздко. Оно показывает, что для произвольного линейно независимого набора векторов $A_{n}$, удовлетворяющего условиям (5), система (70), (71) допускает только тривиальное решение $y_{k} \equiv 0$. Нам представляется, что аналогичное утверждение выполняется при произвольном $N$, но строгое доказательство этого факта пока отсутствует.

Система уравнений (70) и (72) обычно имеет решение, поскольку определитель этих уравнений, как правило, не обращается в нуль. Это решение легко находится при $N=3$, и в конце данного раздела мы покажем, что при определенных условиях оно определяет модули, удовлетворяющие неравенству (68). Мы не рассматриваем общий случай $N>3$. Хотя решение неоднородной линейной системы уравнений (70), (72) может быть легко найдено, задача аналитического вывода общих ограничений на $a_{n}, a_{m n}$, при которых решение удовлетворяет условию (68), оказывается достаточно трудной. 
Поэтому рассмотрим сначала несколько более простую задачу построения решений системы (70), которые удовлетворяют неравенству (68). Предположим (без потери общности), что не все из $a_{m N}$ обращаются в нуль и что квадратная матрица $a_{m k}(2 \leqslant k<N-1,3 \leqslant m<N)$ невырожденна. Тогда, вообще говоря, можно решить неоднородную систему

$$
\sum_{k=2}^{N-1} a_{m k} y_{k}=-a_{m N} y_{N}, \quad m=3, \ldots, N,
$$

и выразить $y_{k}, k=2, \ldots, N-1$, через более или менее произвольно выбранное $y_{N}$ и параметры системы (разумеется, можно выбрать вместо $y_{N}$ любую из переменных $\left.y_{k}\right)$. Соответствующие параметры $\lambda_{n}$ имеют вид

$$
\lambda_{1}=1, \quad \lambda_{k}=1+\frac{y_{k}}{\gamma_{k}}, \quad k=2, \ldots, N .
$$

В силу (68) некоторые из $y_{k}$ обязаны быть отрицательными (если $y_{k}<0$ при всех $k$, то, очевидно, $\lambda_{+}^{2}<1$, так как $\left.\gamma_{k}>0\right)$. Более того, так как

$$
\hat{\lambda}_{+}^{2}=\frac{1}{\left|\gamma_{1}\right|} \sum_{k=2}^{N} \gamma_{k} \lambda_{k}^{2}=\frac{1}{\left|\gamma_{1}\right|} \sum_{k=2}^{N} \gamma_{k}\left(1+\frac{y_{k}}{\gamma_{k}}\right)^{2}=1+\frac{1}{\left|\gamma_{1}\right|} \sum_{k=2}^{N}\left(\frac{y_{k}^{2}}{\gamma_{k}}+2 y_{k}\right) \text {, }
$$

то параметры $y_{k}$ должны удовлетворять достаточно обременительному неравенству

$$
\sum_{k=2}^{N}\left(\frac{y_{k}^{2}}{\gamma_{k}}+2 y_{k}\right)<0
$$

Как следует из предыдущих рассуждений, это неравенство эквивалентно фундаментальным ограничениям (47) и не зависит ни от каких уравнений на $y_{k}$. Модули $\lambda_{n}$, связанные с $y_{k}$ формулами (74) и задающие произвольное решение $(52)\left(\bar{\lambda}_{n}\right.$ определяются формулами (66) (67)), должны удовлетворять этому неравенству.

Возвращаясь к системе (73), заметим, что, подставляя в (76) ее решение, получим квадратное неравенство на произвольный параметр $y_{N}$, но неявная зависимость этого простого неравенства от параметров модели $a_{m n}$ оказывается настолько сложной, что становится практически невозможным сделать какое-либо общее утверждение относительно решения этой задачи ${ }^{16)}$. Мы можем видеть это даже в случае $N=3$. Модель задается тремя произвольными параметрами $a_{31}, a_{32}, a_{33}$. Другие параметры $\left(a_{n}, \gamma_{n}\right)$ можно найти, применяя условия псевдоортогональности. В этом случае имеется лишь одно уравнение (70), которое дает

$$
y_{2}=-\frac{a_{33}}{a_{32}} y_{3} .
$$

${ }^{16)}$ Нетрудно найти решение для произвольной псевдоортогональной системы параметров $a_{m n}$. Однако найти общее аналитическое решение этих уравнений вряд ли возможно. 
Теперь, если $a_{33} / a_{32}<0$, то выберем $y_{3}<0$, что автоматически дает $y_{2}<0$, а потому $\hat{\lambda}_{+}^{2}<1$. Чтобы найти общее решение, рассмотрим кривую в плоскости $\left(\lambda_{2}, \lambda_{3}\right)$, задаваемую условиями $\hat{\lambda}_{+}^{2}=1$, т.е.

$$
\hat{\lambda}_{+}^{2} \equiv \hat{\gamma}_{2}^{2} \lambda_{2}^{2}+\hat{\gamma}_{3}^{2} \lambda_{3}^{2}=1
$$

Так как оба параметра $\lambda_{2}$ и $\lambda_{3}$ зависят только от $y_{3}$, они связаны уравнением, получаемым при исключении этой зависимости ${ }^{17}$

$$
\lambda_{2} a_{32} \hat{\gamma}_{2}^{2}+\lambda_{3} a_{33} \hat{\gamma}_{3}^{2}=a_{31}
$$

Точки $\left(\lambda_{2}, \lambda_{3}\right), \lambda_{2}>0, \lambda_{3}>0$, которые попадают внутрь эллипса (78) и лежат на прямой (79), задают все векторы $\hat{\lambda}_{+}$, удовлетворяющие требуемым условиям. Чтобы найти все возможные значения $\mu_{n}, \nu_{n}$, отвечающие такому вектору, выберем все $\hat{\lambda}_{-}$, ортогональные $\hat{\lambda}_{+}$, с нормой $\hat{\lambda}_{-}^{2}=1-\hat{\lambda}_{+}^{2}$ и таким способом найдем $\bar{\lambda}_{n}$. Решение, полученное для $N$-лиувиллевой задачи при этом задает асимптотически конечные поля скалярной материи, как было объяснено ранее.

Ранее также было отмечено, что представляется возможным найти решение уравнений (70), (72), удовлетворяющее ограничению (68), но это неравенство налагает на параметры $a_{m n}$ определенные условия. Это утверждение легко проиллюстрировать, если рассмотреть простейший нетривиальный случай $N=3$. Вводя обозначение $a_{3 i} \equiv \alpha_{i}$ и напомнив, что (см. [16] и приложение к настоящей работе)

$$
a_{i}=\alpha_{i}\left(\alpha_{j}+\alpha_{k}\right)-\alpha_{j} \alpha_{k}, \quad(i j k)=(123)_{\text {cyclic }}
$$

находим решение

$$
y_{2}=\frac{\alpha_{3}}{8 a_{1}\left(\alpha_{2}-\alpha_{3}\right)}, \quad y_{3}=-\frac{\alpha_{2}}{8 a_{1}\left(\alpha_{2}-\alpha_{3}\right)}
$$

Подставив выражения для $\gamma_{i}$, приведенные в работе [16] (см. также приложение к настоящей работе), можно показать, что $y_{2} / \gamma_{2}$ и $y_{3} / \gamma_{3}$ отрицательны, если $\alpha_{2}<$ $\alpha_{1}<\alpha_{3}, \alpha_{2}<0, \alpha_{3}>0$ (или если $\alpha_{3}<\alpha_{1}<\alpha_{2}, \alpha_{3}<0, \alpha_{2}>0$ ). Отсюда следует, что неравенство $\lambda_{+}^{2}<1$ выполнено, и мы получаем решение, удовлетворяющее всем необходимым условиям. В специальном случае $N=3$, исследуемом в приложении, имеем $a_{1}=-a_{2}=-a_{3}=a, \alpha_{1}=0, \alpha_{2}=\alpha<0, \alpha_{3}=-4 a / \alpha>0$ (если $\left.a>0\right)$. Простые вычисления приводят к ответу $y_{2} / \gamma_{2}=y_{3} / \gamma_{3}=-1 / 2$, а потому $\lambda_{+}^{2}=1 / 4$. Более общие случаи можно рассмотреть аналогично.

Алгоритм построения несингулярных решений при $N \geqslant 4$ принципиально понятен, но на практике оказывается невозможным получить какие-либо достаточно общие утверждения в случае произвольных параметров $a_{m n}$. Как только параметры известны, можно исследовать асимптотические свойства этих решений. Однако

17) Чтобы это получить, необходимо вспомнить тождества (57) и определение $\hat{\gamma}_{k}=\sqrt{\gamma_{k} /\left|\gamma_{1}\right|}$, введенное выше. 
неясно, можно ли найти какую-либо разумную классификацию решений. То же можно сказать и о возможном асимптотическом поведении кривизны $R$. Сингулярная часть кривизны имеет вид

$$
R^{\infty}=\exp \left[-2|\tau|\left(2 \sum_{k=2}^{N} a_{k} y_{k}+\frac{y_{\bar{n}}}{\gamma_{\bar{n}}}\right)\right],
$$

где $\bar{n}$ - номер минимального $\lambda_{n}$, т.е. $\lambda_{\bar{n}}=\lambda_{\min }$ (заметим, что $y_{\bar{n}}$ с необходимостью должно быть отрицательным). В простейшем случае $N=3$ можно записать явное выражение для множителя в круглых скобках в терминах трех свободных параметров $a_{31}, a_{32}, a_{33}$ и тем самым найти, когда $R^{\infty}$ равно нулю, бесконечности или остается конечным; последнее возможно, только если имеет место дополнительное соотношение между этими тремя параметрами (представляющее собой квадратное уравнение на $\left.a_{31}\right)$.

\section{5. РАЗДЕЛЕНИЕ ПЕРЕМЕННЫХ: ПРОСТЫЕ ПРИМЕРЫ}

Построим решения с постоянными модулями, не пользуясь явными решениями в размерности $1+1$. Вместо этого будем применять метод обобщенного разделения переменных. Попробуем сначала разделить переменные $r$ и $t$ в уравнении Лиувилля (19). Если просто записать $r=u+v, t=u-v$ при всех $n$, то, очевидно, мы ограничимся одной из наивных редукций, для которой $q_{n}=q_{n}(r)$ или $q_{n}=q_{n}(t)$.

Однако, учитывая наш опыт работы с решениями $N$-лиувиллевой модели, мы можем попытаться использовать менее наивный подход и выбрать различные $r_{n}, t_{n}$ при разных $n$, положив

$$
r_{n}=\mu_{n} u+\nu_{n} v, \quad t_{n}=\mu_{n} u-\nu_{n} v .
$$

Обозначая штрихом и точкой производные по $r_{n}$ и $t_{n}$, соответственно, получим

$$
q_{n}^{\prime \prime}-\ddot{q}_{n}=\widetilde{G}_{n} e^{q_{n}}, \quad \widetilde{G}_{n} \equiv \frac{\tilde{g}_{n}}{\mu_{n} \nu_{n}} .
$$

Разделим теперь переменные $r_{n}$ и $t_{n}$, записав

$$
q_{n}=\xi_{n}\left(t_{n}\right)+\eta_{n}\left(r_{n}\right)
$$

а затем возьмем от полученного уравнения производную по $r_{n}$ или по $t_{n}$. При этом сразу получим, что возможны следующие два разделения переменных:

$$
\begin{array}{rlrl}
\eta_{n}^{\prime \prime}\left(r_{n}\right)-C_{n} e^{\eta_{n}\left(r_{n}\right)} & =0, & & C_{n} \equiv \widetilde{G}_{n} e^{\xi_{n}}=\mathrm{const}, \\
\ddot{\xi}_{n}\left(t_{n}\right)-\bar{C}_{n} e^{\xi_{n}\left(t_{n}\right)}=0, & \bar{C}_{n} \equiv \widetilde{G}_{n} e^{\eta_{n}}=\mathrm{const} .
\end{array}
$$

Это разделение задает только половину из решений $N$-лиувиллевой модели с постоянными модулями. Но теперь можно предположить, что такая процедура разделения переменных даст другие результаты, если применить ее к $X_{n}$ вместо $q_{n}$. 
В самом деле, попробуем разделить те же переменные (82) в уравнении (22) с использованием того же анзаца (84) для $X_{n}$ :

$$
X_{n}(u, v)=\xi_{n}\left(t_{n}\right)+\eta_{n}\left(r_{n}\right) .
$$

Дифференцируя полученное уравнение

$$
\left(\xi_{n}\left(t_{n}\right)+\eta_{n}\left(r_{n}\right)\right)\left(\eta_{n}^{\prime \prime}\left(r_{n}\right)-\ddot{\xi}_{n}\left(t_{n}\right)\right)-\eta_{n}^{\prime 2}\left(r_{n}\right)+\dot{\xi}_{n}^{2}\left(t_{n}\right)=-\frac{1}{2} \widetilde{G}_{n}
$$

по $r_{n}$, а затем по $t_{n}$, получим, что

$$
\eta_{n}^{\prime}\left(r_{n}\right) \dddot{\xi}_{n}\left(t_{n}\right)-\eta_{n}^{\prime \prime \prime}\left(r_{n}\right) \dot{\xi}_{n}\left(t_{n}\right)=0
$$

и тем самым $\xi_{n}$ и $\eta_{n}$ удовлетворяют уравнениям

$$
\begin{aligned}
\ddot{\xi}_{n}\left(t_{n}\right)-C_{n} \xi_{n}\left(t_{n}\right) & =A_{n}, \\
\eta_{n}^{\prime \prime}\left(r_{n}\right)-C_{n} \eta_{n}\left(r_{n}\right) & =B_{n},
\end{aligned}
$$

где $A_{n}, B_{n}$ и $C_{n}$ - произвольные константы. Таким образом, получим решения

$$
\begin{aligned}
& \xi_{n}\left(t_{n}\right)=-\frac{A_{n}}{C_{n}}+\widetilde{C}_{n}^{-} \operatorname{ch}\left(\sqrt{C_{n}}\left(t_{n}+\delta_{n}^{-}\right)\right), \\
& \eta_{n}\left(r_{n}\right)=-\frac{B_{n}}{C_{n}}+\widetilde{C}_{n}^{+} \operatorname{ch}\left(\sqrt{C_{n}}\left(r_{n}+\delta_{n}^{+}\right)\right)
\end{aligned}
$$

с произвольными константами интегрирования $\widetilde{C}_{n}^{ \pm}, \delta_{n}^{ \pm}$. Подставляя эти функции в уравнения (88), получим, что эти константы должны удовлетворять уравнениям

$$
C_{n}\left(\left(\widetilde{C}_{n}^{+}\right)^{2}-\left(\widetilde{C}_{n}^{-}\right)^{2}\right)=-\frac{\tilde{g}_{n}}{2 \mu_{n} \nu_{n}}, \quad A_{n}+B_{n}=0 .
$$

Отсюда видно, что $X_{n}$, задаваемые уравнениями (87), (92)-(94), совпадают с (48)-(51), если выбрать $C_{n}=1$ и $\widetilde{C}_{n}^{ \pm}=C_{n}^{ \pm} / \sqrt{\mu_{n} \nu_{n}}$ (или $C_{n}=1 /\left(\mu_{n} \nu_{n}\right)$ и $\widetilde{C}_{n}^{ \pm}=$ $\left.C_{n}^{ \pm}\right)$.

В этих рассуждениях интегрируемость $N$-лиувиллевой модели не использовалась, и можно ожидать, что наш подход может быть применим и к другим нелинейным системам, не обязательно интегрируемым. В самом деле, точно таким же способом мы ранее получили все сферически-симметричные статические состояния и космологии, которые описываются в общем случае неинтегрируемыми уравнениями (см. [17]). Поэтому мы ожидаем, что решения, похожие на решения с постоянными модулями в интегрируемой $N$-лиувиллевой теории, могут также существовать и в неинтегрируемых реалистичных $(1+1)$-мерных теориях. Скорее всего, их можно получить, используя некоторое обобщенное разделение переменных. Мы собираемся провести серьезный анализ такой возможности в последующих работах, а здесь ограничимся только очень простым, почти тривиальным примером. 
Напомним обсуждение "реалистичных" потенциалов в разделе 2 и рассмотрим теперь случай единственного скалярного поля, взаимодействующего с дилатонной гравитацией с потенциалами

$$
V=g \varphi^{1+a} e^{\lambda \psi}, \quad Z=-\varphi
$$

Вводя обозначение $\varphi \equiv e^{\phi}$, запишем основные уравнения движения в виде ${ }^{18)}$

$$
\begin{aligned}
& \partial_{u} \partial_{v} \phi+\partial_{u} \phi \partial_{v} \phi+\varepsilon g e^{F+a \phi+\lambda \psi}=0, \\
& 2 \partial_{u} \partial_{v} \psi+\partial_{u} \phi \partial_{v} \psi+\partial_{v} \phi \partial_{u} \psi-\varepsilon g \lambda e^{F+a \phi+\lambda \psi}=0, \\
& \partial_{i}^{2} \phi+\left(\partial_{i} \phi\right)^{2}-\partial_{i} F \partial_{i} \phi+\left(\partial_{i} \psi\right)^{2}=0, \quad i=u, v .
\end{aligned}
$$

Разделение переменных наиболее удобно проводить, если неизвестные функции удовлетворяют билинейным уравнениям. В нашем примере уравнения (98) билинейны, а еще одно билинейное уравнение можно записать, комбинируя (96) с (97). Положив $\Psi=\psi+\lambda \phi / 2$, получим

$$
2 \partial_{u} \partial_{v} \Psi+\partial_{u} \Psi \partial_{v} \phi+\partial_{v} \Psi \partial_{u} \phi=0
$$

Единственным уравнением, содержащим экспоненту, остается уравнение (96). Мы не будем пытаться полностью исследовать эти уравнения, а вместо этого выпишем их весьма специальное решение

$$
\begin{gathered}
\phi=\alpha u+\beta v, \quad \Psi=\mu(\alpha u-\beta v), \\
F=C_{0}-\alpha u\left(a-\frac{\lambda^{2}}{2}+\mu \lambda\right)-\beta v\left(a-\frac{\lambda^{2}}{2}-\mu \lambda\right),
\end{gathered}
$$

где $\alpha, \beta$ - произвольные параметры, $C_{0}=\ln (-\alpha \beta / g)$ и $\mu^{2} \equiv \lambda^{2} / 4-1-a$.

Положив $r \equiv u+v, t \equiv u-v$, можно переписать эти решения в волноподобном (или импульсно-подобном) виде

$$
\begin{aligned}
\phi & =\frac{\alpha+\beta}{2}\left(r+V_{1} t\right), \\
\Psi & =\frac{1}{2}\left[\left(\mu-\frac{\lambda}{2}\right) \alpha-\left(\mu+\frac{\lambda}{2}\right) \beta\right]\left(r+V_{2} t\right), \\
F & =-\frac{1}{2}\left[\alpha\left(a-\frac{\lambda^{2}}{2}+\lambda \mu\right)+\beta\left(a-\frac{\lambda^{2}}{2}-\lambda \mu\right)\right]\left(r+V_{3} t\right),
\end{aligned}
$$

где скорости импульсов таковы:

$$
\begin{aligned}
& V_{1}=\frac{\alpha-\beta}{2}, \\
& V_{2}=\frac{(\mu-\lambda / 2) \alpha+(\mu+\lambda / 2) \beta}{(\mu-\lambda / 2) \alpha-(\mu+\lambda / 2) \beta},
\end{aligned}
$$

18) Как и везде, здесь используется лагранжиан (1) в координатах СК. 


$$
V_{3}=\frac{\alpha\left(a-\lambda^{2} / 2+\mu \lambda\right)-\beta\left(a-\lambda^{2} / 2-\mu \lambda\right)}{\alpha\left(a-\lambda^{2} / 2+\mu \lambda\right)+\beta\left(a-\lambda^{2} / 2-\mu \lambda\right)} .
$$

Выбирая калибровку $\alpha=\beta$ (случай “статичного” $\phi$ ), получим

$$
V_{1}=0, \quad V_{2}=-\frac{2 \mu}{\lambda}, \quad V_{3}=-\frac{2 \mu}{\lambda}\left(1-\frac{2 a}{\lambda^{2}}\right)^{-1} .
$$

Модель, в которой это решение было найдено, принадлежит к классу двумерных теорий, получаемых при стандартных размерных редукциях из теорий старших размерностей. Видно, что волноподобные решения появляются не только в интегрируемых моделях с $N=1$, но также и в достаточно реалистичных и очевидно неинтегрируемых теориях. Поэтому можно надеяться на то, что связь между статическими, космологическими и волноподобными решениями окажется общим свойством теории гравитации ${ }^{19)}$.

\section{6. ЗАКЛЮЧЕНИЕ И ПЕРСПЕКТИВЫ}

Результаты данной работы можно описать следующим образом. Мы обобщили аналитическое выражение для решения уравнений и связей в $(1+1)$-мерной $N$-лиувиллевой теории (см. (29), (30)). Была введена весьма удобная калибровка (40), в которой особенно отчетливо проявляются основные физические свойства решений, а затем найдено представление для решений в терминах функций модулей $\hat{\xi}(u) \in S^{(N-2)}, \hat{\eta}(v) \in S^{(N-2)}$. Редуцируя пространство модулей на пространство пар точек $\left(\hat{\xi}^{(0)}, \hat{\eta}^{(0)}\right)$, лежащих на сфере $S^{(N-2)}$, мы построили и изучили новый интересный класс решений $N$-лиувиллевой теории (см. (45), (46)). При $\hat{\xi}^{(0)}=\hat{\eta}^{(0)}$ эти решения находят интерпретацию либо как статические состояния, либо как космологии. При $\hat{\xi}^{(0)} \neq \hat{\eta}^{(0)}$ соответствующие решения зависят от обеих переменных $r$ и $t$.

В общем случае решения имеют сингулярности в конечных точках пространствавремени $(t, r)$ и в бесконечности. Тем не менее существует подкласс решений, не имеющих сингулярностей в конечных точках (см. (48), (51)). Решения, лежащие в этом (несингулярном) подклассе, обычно обращаются в бесконечность на пространственной и/или временно́й бесконечности. Однако мы показали, что некоторые из них конечны как при $r \rightarrow \pm \infty$, так и при $t \rightarrow \pm \infty$. Мы явно сформулировали связи, обеспечивающие конечность решений на границах пространства и времени; в данном случае эти связи оказалось легко разрешить. Тем не менее их невозможно разрешить в общем виде, а потому мы не смогли вывести общую формулу для решения этих связей в произвольной $N$-лиувиллевой модели. Таким образом, сформулирован алгоритм решения этой задачи, который проиллюстрирован весьма простым случаем $N=3$.

${ }^{19)}$ В работе [17] было показано, что в случае сферически редуцированной гравитации, взаимодействующей со скалярными полями материи, выполнено соотношение дуальности между статическими состояниями и космологией. 
Решения без сингулярностей в конечных точках обладают конечными плотностями энергии и импульса, и их первые производные имеют вид ступеньки, подобно некоторым топологическим солитонам. Таким образом, они напоминают солитоны, но их энергия не локализована в пространстве, а потому мы избегаем употребления термина "солитон” по отношению к таким решениям. Кривизна $R$ этих решений конечна в конечных точках и может расходиться на бесконечности (это зависит от значений всех параметров, задающих решение). Таким образом, несмотря на свою очевидную простоту решения, задаваемые постоянными модулями, представляют собой достаточно сложные объекты, тесно связанные с геометрией пространства-времени.

Нам представляется возможным, что весьма схожие волноподобные решения можно получить в реалистичных неинтегрируемых теориях. Там их следует искать с помощью процедуры обобщенного разделения переменных. Здесь мы показали, что волновые решения интегрируемой $(1+1)$-мерной теории можно получить без использования понятия о ее интегрируемости, а также привели примеры простейших решений в неинтегрируемой теории.

В будущем мы планируем сосредоточить усилия на выводе волноподобных решений, схожих с найденными в данной работе, в реалистичных неинтегрируемых теориях. Достаточно простыми, но интересными волновыми решениями, которые можно исследовать с применением техники данной работы, оказываются цилиндрические волны [36] и плоские волны [37]. Мы также надеемся найти более тесную связь между статическими состояниями, космологиями и волнами в полностью реалистичных теориях. Некоторые физически значимые соотношения между волнами и черными дырами обсуждались в литературе (см., например, работу [38] и ссылки в ней). Возможная роль гравитационных волн в космологии была также рассмотрена в литературе (см., например, работы [39] и [40]). На первый взгляд наши результаты могут показаться не так уж непосредственно связанными с этими исследованиями, но, поскольку наши результаты оказались концептуально и технически весьма простыми, они могут помочь найти новый систематический подход к более глубокому пониманию этого сложного явления.

\section{ПРИЛОЖЕНИЕ}

В приложении приведены параметры нескольких типичных $N$-лиувиллевых моделей, некоторые из которых можно получить с помощью размерной редукции теорий супергравитации старших размерностей. Построим сначала общую модель с $N=4$. Применяя формулы из приложения к работе [16], можно непосредственно выписать общие выражения для $a_{i}$ и $\gamma_{i}, i=1,2,3$, в общем случае при $N=4$. Вводя обозначения $a_{3 n} \equiv \alpha_{n}, a_{4 n} \equiv \beta_{n}, n=1, \ldots, 4$, получим

$$
\begin{aligned}
4 a_{i} & =\alpha_{i}\left(\alpha_{j}+\alpha_{k}\right)-\alpha_{j} \alpha_{k}+\beta_{i}\left(\beta_{j}+\beta_{k}\right)-\beta_{j} \beta_{k}, \\
\gamma_{i} & =\left(\alpha_{i}-\alpha_{j}\right)\left(\alpha_{i}-\alpha_{k}\right)+\left(\beta_{i}-\beta_{j}\right)\left(\beta_{i}-\beta_{k}\right),
\end{aligned}
$$




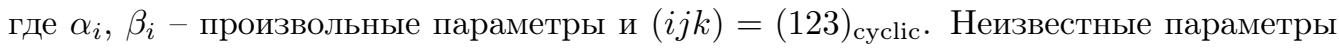
$a_{4}, \alpha_{4}, \beta_{4}$ можно получить, если применить общую процедуру работы [16]. В случае $N=4$ она приводит к трем неоднородным линейным уравнениям на эти три параметра. Решение имеет вид

$$
\begin{aligned}
a_{4} & =-\frac{1}{\Delta}\left[a_{1}\left(\alpha_{2} \beta_{3}-\alpha_{3} \beta_{2}\right)+a_{2}\left(\alpha_{3} \beta_{1}-\alpha_{1} \beta_{3}\right)+a_{3}\left(\alpha_{1} \beta_{2}-\alpha_{2} \beta_{1}\right)\right], \\
\alpha_{4} & =\frac{2}{\Delta}\left[a_{1}\left(\beta_{2}-\beta_{3}\right)+a_{2}\left(\beta_{3}-\beta_{1}\right)+a_{3}\left(\beta_{1}-\beta_{2}\right)\right], \\
\beta_{4} & =-\frac{2}{\Delta}\left[a_{1}\left(\alpha_{2}-\alpha_{3}\right)+a_{2}\left(\alpha_{3}-\alpha_{1}\right)+a_{3}\left(\alpha_{1}-\alpha_{2}\right)\right], \\
\Delta & =\alpha_{1}\left(\beta_{2}-\beta_{3}\right)+\alpha_{2}\left(\beta_{3}-\beta_{1}\right)+\alpha_{3}\left(\beta_{1}-\beta_{2}\right) .
\end{aligned}
$$

Альтернативный способ вывода состоит в том, чтобы применить выражения (П.1), (П.2) и "правила сумм" (57) и получить из этого ответы для $\gamma_{4}, a_{4}, \alpha_{4}, \beta_{4}$ в терминах найденных ранее $a_{i}, \gamma_{i}, i=1,2,3$.

Процедура нахождения параметров модели в случае $N=5$ оказывается гораздо более сложной. Более того, общие выражения оказываются бесполезными для практических аналитических вычислений даже в простых задачах, обсуждаемых в основном тексте статьи. К счастью, реалистичные $N$-лиувиллевы теории, получаемые из теорий старших размерностей, зачастую обладают структурами, более простыми, чем в общем случае. Выше нами приводились некоторые важные примеры:

$$
\begin{aligned}
& -a_{1}=a_{2}=\cdots=a_{N}=-a ; \\
& -a_{1}=-a_{2}=a_{3}=\cdots=a_{N}=-a .
\end{aligned}
$$

Чтобы найти выражения для параметров $a_{m n}$ этих моделей, удобно вместо использования общей процедуры применить простые геометрические соображения. Для этого запишем векторы $A_{n}$, введенные в разделе 2 , в виде

$$
A_{n} \equiv\left(1+a_{n}, 1-a_{n}, \vec{A}_{n}\right), \quad \vec{A}_{n} \equiv\left(a_{3 n}, \ldots, a_{N n}\right),
$$

где $\overrightarrow{A_{n}}$ - евклидовы $(N-2)$-векторы, которые должны удовлетворять ограничениям, вытекающим из уравнений (5). В моделях (П.7) эту задачу можно явно решить при произвольном $N$, но мы здесь выпишем результат только при $N=4$. Для моделей типа (П.7а) имеем

$$
\begin{array}{rlrl}
\vec{A}_{1} & =(0,0), & \gamma_{1}^{-1}=-4 a ; \\
\vec{A}_{2} & =(\alpha, 0), & \gamma_{2}^{-1}=\alpha^{2}+4 a ; \\
\vec{A}_{3}=\left(-\frac{4 a}{\alpha}, \beta\right), & \gamma_{3}^{-1}=\frac{\alpha^{2} \beta^{2}+4 a \alpha^{2}+16 a^{2}}{\alpha^{2}} \\
\vec{A}_{4}=\left(-\frac{4}{\alpha},-\frac{4 a\left(\alpha^{2}+4 a\right)}{\alpha^{2} \beta}\right), & \gamma_{4}=-\left(\gamma_{1}+\gamma_{2}+\gamma_{3}\right) .
\end{array}
$$


Для моделей типа (П.7б) получим

$$
\begin{array}{ll}
\vec{A}_{1}=(\alpha, 0), & \gamma_{1}^{-1}=\alpha^{2}-4 a ; \\
\vec{A}_{2}=\left(\frac{4 a}{\alpha}, 0\right), & \gamma_{2}^{-1}=\frac{4 a\left(4 a-\alpha^{2}\right)}{\alpha^{2}} ; \\
\vec{A}_{3}=(0, \beta) ; & \gamma_{3}^{-1}=\beta^{2}+4 a ; \\
\vec{A}_{4}=\left(0,-\frac{4 a}{\beta}\right), & \gamma_{4}^{-1}=\frac{4 a\left(\beta^{2}+4 a\right)}{\beta^{2}} .
\end{array}
$$

С помощью этих результатов читатель может легко вывести векторы $\vec{A}_{n}$ для моделей типа (П.7) в случае $N=5$ и обобщить эти формулы на случай произвольного $N$.

Примеры (П.7) представляют интерес не только вследствие своей простоты. Как было отмечено в разделе 2, они оказываются общими решениями в контексте размерной редукции некоторых теорий супергравитации старших размерностей (см., например, модель в работе [25], параметры которой приведены в (15)).

Благодарности. А.Т. Филиппов глубоко признателен Отделу теоретической физики Университета г. Турина и INFN (Sezione di Torino) за поддержку. Работа была частично поддержана РФФИ (грант № 06-01-00627-а).

\section{Список литературы}

[1] T. Padmanabhan, AIP Conf. Proc., 861 (2006), 179; astro-ph/0603114.

[2] E. J. Copeland, M. Sami, S. Tsujikawa, Internat. J. Modern Phys. D, 15 (2006), 1753; hep-th/0603057.

[3] S. Nojiri, S. Odintsov, Int. J. Geom. Methods Mod. Phys., 4 (2007), 115; hep-th/0601213.

[4] V. Sahni, A. Starobinsky, Internat. J. Modern Phys. D, 15 (2006), 2105; astro-ph/0610026.

[5] J. E. Lidsey, D. Wands, E. J. Copeland, Phys. Rep., 337 (2000), 343.

[6] M. Gasperini, G. Veneziano, Phys. Rep., 373 (2003), 1.

[7] H. Stefani, D. Kramer, M. MacCallum et al., Exact Solutions of the Einstein's Field Equations, Cambridge Univ. Press, Cambridge, 2002.

[8] В.А.Белинский, В.Е. Захаров, ЖЭТФ, 75:6 (1978), 1955.

[9] D. Maison, Phys. Rev. Lett., 41 (1978), 521.

[10] H. Nicolai, D. Korotkin, H. Samtleben, "Integrable classical and quantum gravity", Quantum Fields and Quantum Space Time (Cargèse, Corsica, France, 1996), NATO Adv. Sci. Inst. Ser. B. Phys., 364, Plenum, New York, 1997, 203; hep-th/9612065.

[11] Г. А. Алексеев, ТМФ, 143:2 (2005), 278.

[12] C. Callan, S. Giddings, J. Harvey, A. Strominger, Phys. Rev. D (3), 45 (1992), R1005.

[13] V. de Alfaro, A. T. Filippov, Integrable low dimensional theories describing higher dimensional branes, black holes, and cosmologies, hep-th/0307269.

[14] V. de Alfaro, A.T. Filippov, Integrable low dimensional models for black holes and cosmologies from high dimensional theories, hep-th/0504101.

[15] V. de Alfaro, A. T. Filippov, "Black holes and cosmological solutions in various dimensions" (unpublished).

[16] А. Т. Филиппов, ТМФ, 146:1 (2006), 115; hep-th/0505060.

[17] A. T. Filippov, Some unusual dimensional reductions of gravity: geometric potentials, separation of variables, and static-cosmological duality, hep-th/0605276. 
[18] V. de Alfaro, A. T. Filippov, "Dynamical dimensional reduction" (unpublished).

[19] A. T. Filippov, Modern Phys. Lett. A, 11 (1996), 1691; Internat. J. Modern Phys. A, 12 (1997), 13.

[20] J. R. Oppenheimer, H. Snyder, Phys. Rev. D, 55 (1939), 374.

[21] M. Cavaglià, V. de Alfaro, A. T. Filippov, Internat. J. Modern Phys. D, 4 (1995), 661; 5 (1996), 227; 6 (1997), 39.

[22] A. Lukas, B. A. Ovrut, D. Waldram, Phys. Lett. B, 393 (1997), 65.

[23] F. Larsen, F. Wilczek, Phys. Rev. D, 55 (1997), 4591.

[24] H. Lü, S. Mukherji, C. N. Pope, Internat. J. Modern Phys. A, 14 (1999), 4121.

[25] Y. Kiem, C. Y. Lee, D. Park, Phys. Rev. D (3), 57 (1998), 2381.

[26] G. D. Dzhordzhadze, A. K. Pogrebkov, M. C. Polivanov, On the solutions with singularities of the Liouville equation, Preprint IC/78/126, ICTP, Trieste, 1978.

[27] J. L. Gervais, Internat. J. Modern Phys. A, 6 (1991), 2805.

[28] А.Н. Лезнов, М.В. Савельев, Групповые методы интегрирования нелинейных динамических систем, Наука, М., 1985.

[29] L. Castellani, A. Ceresole, R. D'Auria, S. Ferrara, P. Fré, M. Trigiante, Nucl. Phys. B, 527 (1998), 142.

[30] P. Fré, A. Sorin, Nucl. Phys. B, 733 (2006), 334.

[31] K. Stelle, "BPS branes in supergravity", Quantum Field Theory: Perspective and Prospective (Les Houches, France, 1998), NATO Sci. Ser. C. Math. Phys. Sci., 530, Kluwer, Dordrecht, 1999, 257; hep-th/9803116.

[32] T. Mohaupt, Class. Quantum Grav., 17 (2000), 3429.

[33] V.D. Ivashchuk, V. N. Melnikov, Class. Quantum Grav., 18 (2001), R87.

[34] Г. П. Джорджадзе, А. К. Погребков, М. К. Поливанов, ТМФ, 40:2 (1979), 221; G. Р. Jorijadze, A. K. Pogrebkov, M. C. Polivanov, J. Phys. A, 19 (1986), 121.

[35] E. D'Hoker, R. Jackiw, Phys. Rev. D (3), 26 (1982), 3517; Phys. Rev. Lett., 50 (1983), 1719.

[36] A. Einstein, N. Rosen, J. Franklin Inst., 223 (1937), 43; N. Rosen, Phys. Z. Zowjetunion, 12 (1937), 366.

[37] P. Szekeres, J. Math. Phys, 13 (1972), 286.

[38] I. Ya. Aref'eva, K. S. Viswanathan, I. V. Volovich, Nucl. Phys. B, 452 (1995), 346.

[39] A. Feinstein, K. E. Kunze, M. A. Vázquez-Mozo, Class. Quantum Grav., 17 (2000), 3599.

[40] V. Bozza, G. Veneziano, JHEP, 10 (2000), 035. 